\title{
Turbulent Transport of Spray Droplets in the Vicinity of Moving Surface Waves
}

\author{
DAVid H. Richter AND ANNE E. DEMPSEY \\ University of Notre Dame, Notre Dame, Indiana \\ Peter P. Sullivan \\ National Center for Atmospheric Research, Boulder, Colorado
}

(Manuscript received 14 January 2019, in final form 23 April 2019)

\begin{abstract}
A common technique for estimating the sea surface generation functions of spray and aerosols is the socalled flux-profile method, where fixed-height concentration measurements are used to infer fluxes at the surface by assuming a form of the concentration profile. At its simplest, this method assumes a balance between spray emission and deposition, and under these conditions the concentration profile follows a powerlaw shape. It is the purpose of this work to evaluate the influence of waves on this power-law theory, as well as investigate its applicability over a range of droplet sizes. Large-eddy simulations combined with Lagrangian droplet tracking are used to resolve the turbulent transport of spray droplets over moving, monochromatic waves at the lower surface. The wave age and the droplet diameter are varied, and it is found that droplets are highly influenced both by their inertia (i.e., their inability to travel exactly with fluid streamlines) and the wave-induced turbulence. Deviations of the vertical concentration profiles from the power-law theory are found at all wave ages and for large droplets. The dynamics of droplets within the wave boundary layer alter their net vertical fluxes, and as a result, estimates of surface emission based on the flux-profile method can yield significant errors. In practice, the resulting implication is that the flux-profile method may unsuitable for large droplets, and the combined effect of inertia and wave-induced turbulence is responsible for the continued spread in their surface source estimates.
\end{abstract}

\section{Introduction}

Sea spray droplets and marine aerosols play several significant roles in Earth's weather and climate, and any attempt to quantify these impacts relies heavily on accurately constraining their production at the ocean surface. In this regard, many studies spanning the last several decades have been devoted to measuring either their surface flux or airborne concentration (Blanchard et al. 1984; Monahan 1968; de Leeuw 1986; Jones and Andreas 2012; Lenain and Melville 2017); however, it has become increasingly clear that a primary source of the large uncertainty which characterizes sea spray droplet and aerosol generation functions (de Leeuw et al. 2011; Veron 2015) results from a substantial lack of insight into the turbulent transport of droplets in the vicinity of surface waves. This transport is likely much more complex than the homogeneous theory that spray

\footnotetext{
Corresponding author: David H. Richter, david.richter.26@ nd.edu
}

source functions are often inferred from, and droplet dynamics are subject to phenomena such as local flow inhomogeneities (e.g., flow separation) and size effects (e.g., droplet inertia). For instance, a common method for estimating surface production of spray and marine aerosols is to use flux-profile relationships (Lewis and Schwartz 2004), but under the assumption of spatial homogeneity, temporal stationarity, or a balance between deposition and emission (Hoppel et al. 2002).

It is well known that through pressure work, surface waves alter the exchange of momentum between the air and the ocean. From the perspective of the air-side boundary layer this can sometimes be accounted for by a modification to the bulk surface roughness (e.g., Edson et al. 2013), but in the immediate vicinity of the waves, relationships such as Monin-Obukhov similarity theory are invalid owing to a near-surface wave boundary layer and modifications/redistributions to the overall momentum flux (Belcher and Hunt 1998; Sullivan and McWilliams 2010; Hara and Sullivan 2015). The influence of waves on the transfer of other quantities, such as air-sea gas exchange 
(Wanninkhof et al. 2009), is also an area of active research. Historically, much work has focused on studying airflow separation past waves and the resulting changes to drag and pressure (Buckles et al. 1984; Banner 1990), and more recent observations are beginning to yield highly detailed insight into the structure of the turbulent airflow around waves (Reul et al. 2008; Buckley and Veron 2016; Tamura et al. 2018).

Likewise, direct numerical simulations (DNS) and large-eddy simulations (LES) are also being leveraged to understand basic processes associated with waveturbulence interaction (De Angelis et al. 1997; Sullivan et al. 2000; Yang and Shen 2010; Druzhinin et al. 2012; Sullivan et al. 2018a,b), and the combination of highresolution simulations and experiments have demonstrated that intermittent airflow separation occurs prior to wave breaking, and that factors such as wave slope and phase speed modulate much of the exchange between the waves and the flow above. Sullivan et al. (2000) and Yang and Shen (2010) used DNS to study turbulence over idealized, monochromatic waves in planar Couette flow, and later Sullivan et al. (2008), Sullivan et al. (2014), and Sullivan et al. (2018b) developed an LES model capable of resolving turbulence over transient, nonmonochromatic waves at the lower boundary. Detailed momentum flux budgets were constructed in order to observe the impact of pressure work on drag (see also Hara and Sullivan 2015), and fundamental differences between momentum and scalar transport over wavy surfaces were identified. While the wavy surfaces in these DNS and LES studies were prescribed, other numerical methods have been used which dynamically capture the formation of capillary and surface gravity waves, and/or their breaking.

Furthermore, advanced numerical and experimental methods are helping shed light on near-surface droplet behavior in wavy or high-wind conditions. For instance, Tang et al. (2017) coupled an interface-tracking numerical scheme to a Lagrangian droplet tracking method in order to investigate droplet dynamics near breaking waves. Other studies, such as Derakhti and Kirby (2014) and Deike et al. (2016) have used similar approaches to study air entrainment and bubble transport on the water side during breaking events. In the laboratory, highresolution imaging has yielded information on droplet formation mechanisms (Veron et al. 2012; Troitskaya et al. 2017), and wind tunnels capable of achieving high winds (albeit with very young waves) have improved our understanding of the production and suspension of spume droplets (Fairall et al. 2009; Ortiz-Suslow et al. 2016; Komori et al. 2018). It is becoming increasingly clear that large droplets are confined to the vicinity of the wave and often evade detection by elevated fixed-height measurements (Veron 2015); however, it remains uncertain how quickly the droplet concentration decreases with height, and how it varies with droplet radius. Traditional equilibrium models, such as those by Rouse (1937) or Prandtl (1952) predicting a power-law decrease in concentration with height, are almost certainly inaccurate in the wave boundary layer. Since spume droplets carry the highest potential for altering air-sea momentum, heat, and moisture transfer (Andreas et al. 1995), it is important to understand their fate as they are carried through a turbulent airflow.

In the present study, we are primarily interested in the influence of waves on the transport of droplets. As noted in Sullivan et al. (2018b), the additional mechanism of pressure work for transporting momentum across the air-water surface does not exist for scalar transport (i.e., there is no analogous pressure transport of scalars), and thus any impact of waves is indirect - the waves alter the turbulence, which in turn modifies turbulent fluxes. Since the same is true for droplet transport, and since near-surface distributions of spray remain almost completely unknown in high-wind conditions, we aim to analyze near-wave distributions of droplets over a range of wave age and droplet size. In particular, a primary objective is to assess the accuracy of equilibrium theories such as that of Prandtl (1952) which are frequently used in inferring sea surface generation functions for spray and marine aerosols. To achieve this goal, we combine the LES model of Sullivan et al. (2018b) with a Lagrangian treatment of spray droplets as implemented, for example, in Richter and Sullivan (2013) and Peng and Richter (2017). The use of Lagrangian droplets coupled to an Eulerian flow field is an increasingly popular method for studying particle-turbulence interaction (Balachandar and Eaton 2010), and has been used in the past for investigating wavy surfaces as well (Marchioli et al. 2006; Druzhinin et al. 2017). Because droplets behave inertially near the surface (i.e., they do not travel with fluid streamlines), treating them from a Lagrangian point of view is advantageous for the purposes of this study and yields useful information for parameterizing droplet production at the wavy ocean surface.

\section{Numerical methodology}

The numerical methodology is similar to that of Druzhinin et al. (2017) and Druzhinin et al. (2018) and builds on the previous work of Richter and Chamecki (2018) and Sullivan et al. (2018b). The strategy is to use LES for simulating the turbulent airflow over prescribed surface waves and to track the trajectories of Lagrangian droplets after they are ejected from the lower surface. In 
this study we focus entirely on the transport and suspension of these droplets (i.e., one-way coupling), and defer discussion of two-way coupling-where droplets can exchange momentum, heat, and/or vapor mass with the surrounding air-to future work.

\section{a. LES of the air phase}

For the Eulerian air phase, the LES code solves the filtered equations for mass and momentum conservation in a transient, nonorthogononal, surface-following coordinate system. The physical, Cartesian coordinates are given by $x_{i}=(x, y, z)$, and these are mapped into a rectangular computational domain $\xi_{i}=(\xi, \eta, \zeta)$ based on the surface wave height $h(x, t)$, which varies in time and streamwise distance (spanwise variations of $h$ are not included in this study). In this code the streamwise and spanwise coordinates are kept constant during the transformation: $\xi=x$ and $\eta=y$. Additional details of the coordinate transformation and grid stretching scheme are provided in Sullivan et al. (2018b), but we note here that the wave, and therefore the grid, is transient in time according to the prescribed moving surface height (to be detailed below), and therefore an accurate numerical implementation requires additional considerations beyond the traditional configuration of flow past stationary bumps.

In this coordinate transformation the contravariant velocity $U_{i}$, which is the cell-edge flux velocity perpendicular to the grid cell in the computational domain, is calculated based on the Cartesian velocity $u_{i}$ in physical space as

$$
U_{i}=\frac{u_{j}}{J} \frac{\partial \xi_{i}}{\partial x_{j}}
$$

where $J$ is the Jacobian of the grid transformation. With this defined, the equations for mass and momentum conservation are given by

$$
\frac{\partial U_{i}}{\partial x_{i}}=0
$$

and

$$
\begin{aligned}
\frac{\partial}{\partial t}\left(\frac{u_{i}}{J}\right)+\frac{\partial}{\partial \xi_{j}}\left[\left(U_{j}-z_{t} \delta_{3 j}\right) u_{i}\right]= & -\frac{\partial}{\partial \xi_{j}}\left(\frac{p}{J} \frac{\partial \xi_{j}}{\partial x_{i}}\right) \\
& -\frac{\partial}{\partial \xi_{j}}\left(\frac{\tau_{i k}}{J} \frac{\partial \xi_{j}}{\partial x_{k}}\right)-\frac{1}{J} \frac{\partial \mathscr{P}}{\partial x} \delta_{1 i}
\end{aligned}
$$

where $p$ is the pressure, $\partial \mathscr{P} / \partial x$ is a constant applied streamwise pressure gradient that drives the flow, $z_{t}$ is the grid translation speed in the $z$ direction, and $\tau_{i k}$ is the subgrid stress, whose form is the same as that used by Moeng (1984) and Sullivan et al. (1996) (among others), and is not repeated here. The flow is assumed neutral and thus no buoyancy term is included in Eq. (3).

In the computational domain, the boundary conditions in the streamwise $\xi$ and spanwise $\eta$ directions are periodic, and thus a pseudospectral discretization is used for spatial gradients. In the wall-normal direction $\zeta$, second-order finite differences are used to approximate vertical derivatives. At the top boundary $\zeta=z=L_{z}$, a no-stress condition is applied to the flow. At the lower wavy boundary $\zeta=0$, a rough wall boundary condition based on Monin-Obukhov similarity theory is implemented locally in space (i.e., on a point-by-point basis rather than on horizontally averaged quantities; Sullivan et al. 2014). Time integration is done via a third-order Runge-Kutta scheme, and incompressibility is enforced by solving a Poisson equation for the pressure. Throughout the study, the time step is held fixed at $\Delta t / \tau_{f}=4 \times 10^{-4}$, where $\tau_{f}=L_{z} / u_{*}(u *$ is the friction velocity based on the total stress at the surface). Unless otherwise noted, a grid of size $\left[N_{x}, N_{y}, N_{z}\right]=[128,128,128]$ is used over a domain size of $\left[L_{x}, L_{y}, L_{z}\right]=[6 \lambda, 5 \lambda, \lambda]$, where $\lambda$ is the wavelength of the imposed lower surface wave.

\section{b. Lagrangian treatment of droplets}

In addition to the solution of Eqs. (2) and (3) for the filtered pressure and velocity fields, the trajectories of individual droplets are solved in a Lagrangian frame of reference. Each droplet moves according to its own velocity:

$$
\frac{d x_{p, i}}{d t}=v_{p, i}
$$

where $x_{p, i}$ is the droplet location in physical space and $v_{i, p}$ is the droplet velocity. Momentum conservation applied to the droplet provides the governing equation for the droplet velocity:

$$
\frac{d v_{p, i}}{d t}=\frac{f}{\tau_{p}}\left(u_{f, i}-v_{p, i}\right)-g \delta_{3 i},
$$

where $\tau_{p}=\rho_{p} d_{p}^{2} /\left(18 \nu_{a}\right)$ is the droplet acceleration time scale, $f=1+0.15 \mathrm{Re}_{p}^{0.687}$ is a correction factor to the hydrodynamic Stokes drag based on the droplet Reynolds number $\operatorname{Re}_{p}=\left|u_{f, i}-v_{p, i}\right| d_{p} / \nu_{a}, d_{p}$ is the droplet diameter, $\rho_{p}$ is the droplet density, $\nu_{a}$ is the kinematic viscosity of the air, $u_{f, i}$ is the filtered velocity interpolated from the computational mesh to the droplet location using sixth-order Lagrange interpolation, and $g$ is the magnitude of the acceleration of gravity. In this setup, we neglect the influence of subgrid velocity fluctuations on the droplet motion since 1) for the droplet 
sizes under consideration inertia effectively acts as a lowpass filter, eliminating these fluctuations [the Stokes number based on the Kolmogorov time scale $\tau_{K}, \mathrm{St}=$ $\tau_{p} / \tau_{K}$, is $O(10)$ even for the smallest diameter considered], and 2) the subgrid stresses are overwhelmed by resolved stresses except at the first few grid points near the wall. Tests have been performed using the subgrid model of Weil et al. (2004) with little impact on the droplet motion. Since our primary focus is on droplet transport and dispersion in the wave boundary layer, we turn off evaporation and heat transfer in this study, noting that it is relatively straightforward to include (Helgans and Richter 2016; Peng and Richter 2017).

In all simulations presented, a monodisperse collection of droplets is initiated randomly throughout the domain into an already-established, statistically steady turbulent field, and from that point forward, the total number of droplets is held fixed at $N_{p}=5 \times 10^{5}$. At the top boundary $\zeta=z=L_{z}$, droplets rebound elastically (equivalent to a no-flux condition for droplet concentration), while at the bottom boundary, a new droplet is introduced every time a droplet crosses $\zeta=0$. Each new droplet has the same diameter and is given a uniformly random horizontal position along the lower boundary $\zeta=0$, intentionally choosing to not produce more droplets at any wave-specific location. This idealized droplet ejection scenario will emphasize the bulk turbulent transport, without arbitrarily biasing our results based on speculation about where droplets should be forming on the wave surface.

Likewise, the droplets are all ejected with an initial velocity equal to the local wave orbital velocity, plus a random vertical velocity component distributed uniformly between 0 and $V_{p, \text { max }}$, where $V_{p \text {,max }}$ is a function of droplet radius and chosen according to the simple constraint that the Reynolds number $\operatorname{Re}_{0}=d_{p} V_{p, \max } / \nu_{a}=140$ is a constant [see, e.g., the discussion in section 4.3.2.3 of Lewis and Schwartz (2004)]. This relationship is based on laboratory measurements of jet droplets (as opposed to film or spume droplets), but in the absence of reliable data on the initial velocities of spume droplets, we consider this an appropriate estimate for our purposes. This is a similar approach as that taken by Edson and Fairall (1994). While varying nonmonotonically with radius, the maximum height any droplet can reach in the absence of turbulent transport is $\zeta / L_{z} \equiv H_{d}=0.36$. As laboratory experiments continue to improve their ability to investigate and quantify droplet formation processes at the water surface (Veron et al. 2012; Troitskaya et al. 2018a,b), we plan to update the numerical droplet initiation as more reliable location and velocity data become available.

Equations (4) and (5) are integrated in time using the same third-order Runge-Kutta scheme as used for the
Eulerian flow. The droplet position in physical space $\left(x_{p, i}\right)$ must be transformed to computational space for the interpolation of the flow velocity, and a NewtonRaphson technique is utilized for this purpose. As presented in the following sections, droplet statistics are collected both from an Eulerian and Lagrangian point of view. Eulerian statistics are calculated by averaging various droplet attributes (number, velocity) in the Eulerian grid volumes within computational space, while Lagrangian statistics are calculated by tracking droplet-specific quantities (lifetime, maximum height achieved) for all individual droplets in the flow.

\section{c. Numerical experiment}

Throughout this study, a moving, monochromatic wave is prescribed at the lower boundary, given by

$$
h(x, t)=A \cos \left[\frac{2 \pi}{\lambda}(x-c t)\right],
$$

where $\lambda$ is the wavelength, $c$ is the phase speed determined by the deep-water dispersion relation $c^{2}=\lambda g /(2 \pi)$, and $A$ is the wave amplitude. The associated wavenumber is $k=2 \pi / \lambda$, and resulting wave slope is simply $A k$. The wave slope in this study is held fixed at $A k=0.2$. From linear wave theory, the corresponding orbital velocities $u_{o}$ and $w_{o}$ at the wave surface are given by

$$
u_{o}=\frac{2 \pi A c}{\lambda} \cos \left[\frac{2 \pi}{\lambda}(x-c t)\right]
$$

and

$$
w_{o}=-\frac{2 \pi A c}{\lambda} \sin \left[\frac{2 \pi}{\lambda}(x-c t)\right] .
$$

The orbital velocities are used in the application of the lower air boundary conditions, as well as in calculating the initial droplet velocities at injection.

Our ultimate goal is to describe droplet transport in the wave boundary layer, and we focus primarily on two key parameters: droplet size and wave age. The size of the droplet controls its inertia as well as its gravitational settling, and we aim to understand the similarities and differences between large and small droplets produced at the water surface. Wave age, on the other hand, can lead to wide variations in the air turbulence given the same bulk (e.g., $10 \mathrm{~m}$ ) velocity, and thus potentially droplet transport as well. While laboratory studies such as Buckley and Veron (2016) and Ortiz-Suslow et al. (2016) are typically limited to very young waves, numerical simulations of older waves and swell indicate that momentum fluxes and vertical velocity fluctuations can be significantly modified by wave age (Sullivan et al. 2000; Jiang et al. 2016). One of 
the goals of this study is to understand the impact this modification has on droplet transport.

The suite of simulations performed is based on a nominal case chosen to lie within a range of dimensional parameters realistically achieved in a laboratory windwave tank: $\lambda=0.5 \mathrm{~m}, c=0.883 \mathrm{~m} \mathrm{~s}^{-1}, d_{p}=100 \mu \mathrm{m}$, and $u_{*}=0.883 \mathrm{~m} \mathrm{~s}^{-1}$, where $u_{*}$ is directly related to the applied pressure gradient: $L_{z} d \mathscr{P} / d x=\rho_{a} u_{*}^{2}\left(\rho_{a}\right.$ is the density of the air). This value of $u_{*}$ corresponds to a simulated maximum velocity of $U_{\max }=13.7 \mathrm{~m} \mathrm{~s}^{-1}$ at the top of the domain.

In the first set of simulations, this baseline turbulent flow is held fixed, and the droplet diameter $d_{p}$ is systematically varied. In the second set of simulations, we vary the dimensionless wave age $c / u_{*}$ by keeping the wave attributes constant and varying $u *$ via $d \mathscr{P} / d x$. For these simulations, rather than simply hold the dimensional droplet diameter $d_{p}$ constant, we aim to ensure that we are isolating the effects of wave age on droplet transport. Thus, while holding $d_{p}$ constant we also modify $\rho_{p}$ and the acceleration of gravity $g$ so as to maintain a constant droplet Stokes number and dimensionless settling velocity. The dimensionless Stokes number indicates the importance of droplet inertia and is defined by $\mathrm{St}=\tau_{p} / \tau_{f}$, where $\tau_{f}=L_{z} / u_{*}$. The dimensionless settling velocity determines the droplet settling tendency relative to the strength of the turbulence and is given by $w_{s} / u_{*}$, where $w_{s}=\tau_{p} g$ is the still-air Stokes settling velocity of a sphere. With these held fixed, any changes in droplet transport among the various values of $c / u_{*}$ are due entirely to changes in the wave boundary layer turbulence since these dimensionless quantities dictate droplet-turbulence interaction. The final set of simulations is provided in Table 1.

\section{Results}

\section{a. Flow statistics}

We begin by briefly presenting a statistical description of the airflow, demonstrating that the turbulence exhibits the similar features as observed in other experimental and numerical studies of wind over surface waves. Throughout the next sections, we use $\langle\cdot\rangle$ to indicate full horizontal and temporal averages in computational space (which are only a function of $\zeta$ ), $\overline{(\cdot)}$ to indicate full horizontal and temporal averages in physical space (only a function of $z$ ), and [.] to indicate temporal and phase averages (which are a function of both $z$ and $x$ ) and which provide information about the wave-correlated statistics.

Figure 1 presents vertical profiles of mean velocity, streamwise and vertical velocity fluctuations, and stress as a function of $\zeta$ for the various wave ages. Figure 1a
TABLE 1. Parameters for the simulations presented in this study. Case names beginning with "d" refer to cases with varying diameter, and case names beginning with "c" refer to cases with varying $c / u_{*}$.

\begin{tabular}{lcccccl}
\hline \hline & Case & $u *\left(\mathrm{~m} \mathrm{~s}^{-1}\right)$ & $c / u *$ & $d_{p}(\mu \mathrm{m})$ & \multicolumn{1}{c}{$\mathrm{St}$} & $w_{s} / u_{*}$ \\
\hline Vary $d_{p}$ & $\mathrm{~d} 10$ & 0.883 & 1 & 10 & 0.0006 & 0.0039 \\
& $\mathrm{~d} 20$ & 0.883 & 1 & 20 & 0.0025 & 0.016 \\
& $\mathrm{~d} 50$ & 0.883 & 1 & 50 & 0.016 & 0.098 \\
& $\mathrm{~d} 100$ & 0.883 & 1 & 100 & 0.063 & 0.39 \\
& $\mathrm{~d} 200$ & 0.883 & 1 & 200 & 0.25 & 1.57 \\
Vary $c / u_{*}$ & $\mathrm{~d} 600$ & 0.883 & 1 & 600 & 2.25 & 14.1 \\
& $\mathrm{c} 1$ & 0.883 & 1 & 100 & 0.063 & 0.39 \\
& $\mathrm{c} 5$ & 0.177 & 5 & 100 & 0.063 & 0.39 \\
Flat & $\mathrm{c} 10$ & 0.088 & 10 & 100 & 0.063 & 0.39 \\
& $\mathrm{f} 1$ & 0.883 & 0 & 100 & 0.063 & 0.39 \\
\hline
\end{tabular}

illustrates the well-known behavior that the wavy surface shifts the mean velocity due to the extra stress at the surface (i.e., a larger equivalent roughness length), and shows only small differences between cases c1, c5, and $\mathrm{c} 10$. The wave ages investigated here do not yet approach the swell regime, where momentum fluxes can be upward and can accelerate the mean velocity (Grachev and Fairall 2001). The streamwise velocity fluctuations $\left\langle u^{\prime 2}\right\rangle$ decrease with wave age, beginning with a near-surface peak which is nearly 2 times larger than the corresponding flat case (Fig. 1b). The vertical velocity fluctuations $\left\langle w^{2}\right\rangle$ also shift as a function of wave age, but particularly as $c / u *$ approaches a value of 10 . These statistics are generally consistent with previous simulations (Sullivan et al. 2000; Druzhinin et al. 2012; Jiang et al. 2016; Sullivan et al. 2018a) and experimental observations (Banner 1990; Buckley and Veron 2016). Regarding the momentum flux, Fig. 1d shows that in the presence of waves, the wavecorrelated motions induce a form drag which is a substantial portion of the total drag near the surface. This component of the drag is largest for the lowest wave age, and the altered turbulence results in a nonmonotonic decrease of the turbulent stress near the center of the domain with increasing $c / u *$. Again, these features are entirely consistent with current understanding of drag and stress over waves (Kudryavtsev and Makin 2001; Edson et al. 2013; Hara and Sullivan 2015; Sullivan et al. 2018b).

In addition to Fig. 1 for characterizing the turbulent flow, Fig. 2 presents the normalized phase-averaged vertical velocity $[w] / u *$. At low wave age, the upstream side of the wave induces a positive $[w]$ as the flow is deflected upward, followed by a negative $[w]$ on the lee side. As $c / u_{*}$ is increased, these perturbations shift phase, and at $c / u_{*}=10$ (Fig. 2c), the clear emergence of a critical layer is observed (Miles 1957), exhibiting a pattern of $[w]$ which again has been observed in simulations 
(a)

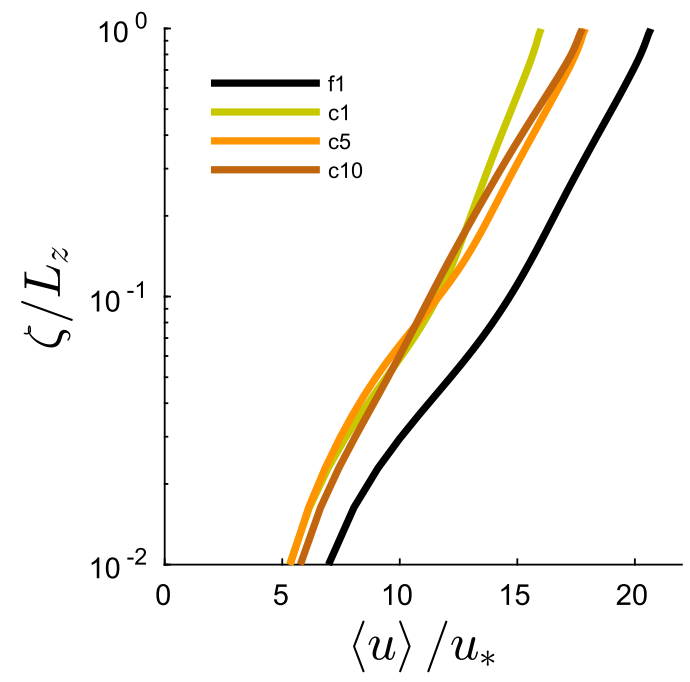

(c)

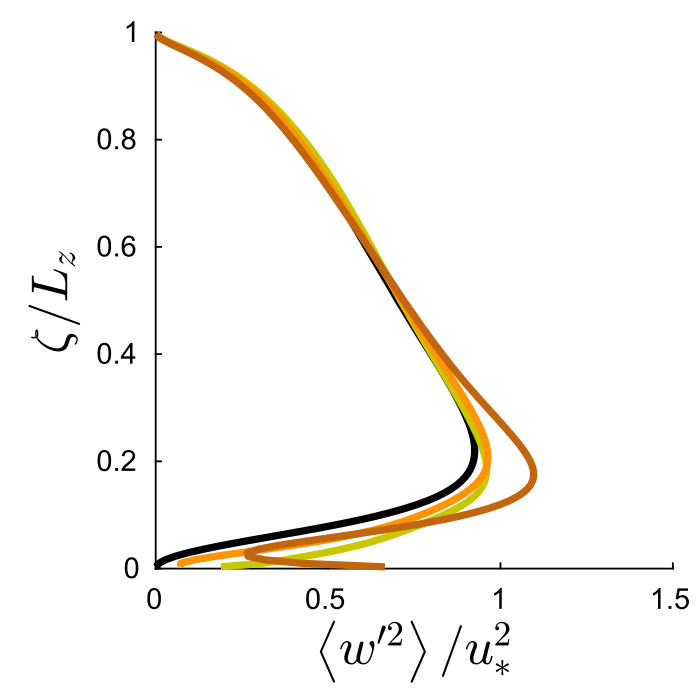

(b)

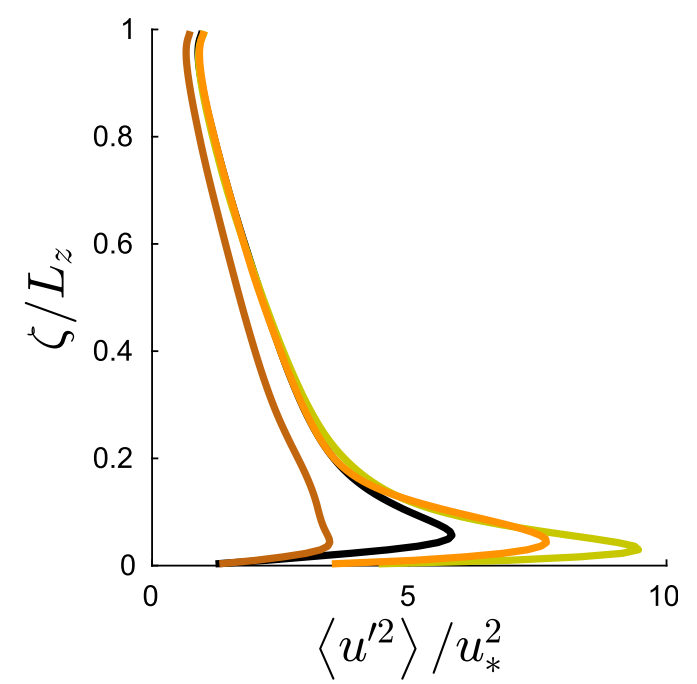

(d)

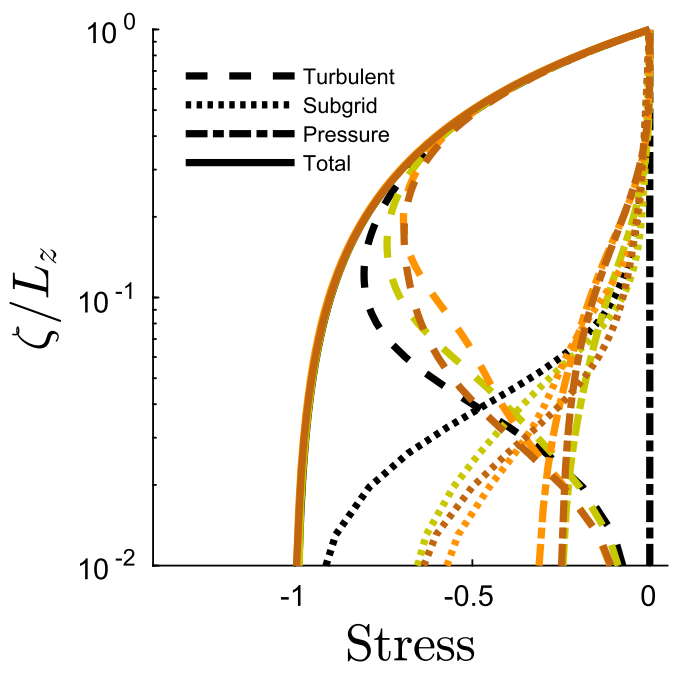

FIG. 1. Statistics of the air turbulence for the various wave ages as a function of the computational vertical coordinate $\zeta$. (a) Mean velocity $\langle u(z)\rangle$, (b) streamwise velocity fluctuation $\left\langle u^{2}\right\rangle$, (c) vertical velocity fluctuation $\left\langle w^{2}\right\rangle$, and (d) stress components (turbulent, wave-pressure correlation, and subgrid; see Sullivan et al. 2018b) normalized by $\rho_{a} u_{*}^{2}$.

(Sullivan et al. 2000) and measurements (Hristov et al. 2003; Grare et al. 2013). This transition of the wavecorrelated vertical velocity field with wave age, whose influence extends for nearly half a wavelength above the surface, indicates a significant change in the flow within the wave boundary layer, and a primary goal of the present study is to investigate the degree to which this modifies the transport and distribution of spray droplets.

\section{b. Droplet statistics: Effect of droplet size}

For the turbulent flow described above, spray droplets are introduced and their transport is quantified for varying droplet size and for varying wave age. In this section we focus first on the effects of spray size on their spatial distribution and lifetime. Figure 3 provides a representative snapshot of $d_{p}=100 \mu \mathrm{m}$ droplets in turbulent flow at a wave age of $c / u_{*}=1$ (i.e., case $\mathrm{c} 1$ ). Qualitative evidence of droplet clustering can be observed, where inertia causes droplets to accumulate in high-strain, low-vorticity regions of the flow (Balachandar and Eaton 2010). Likewise, the intermittent and instantaneously heterogeneous flow separation seen in the experiments of Reul et al. (2008) and Buckley and Veron (2016) can be observed as well. Simulations, such as the DNS of 
(a)

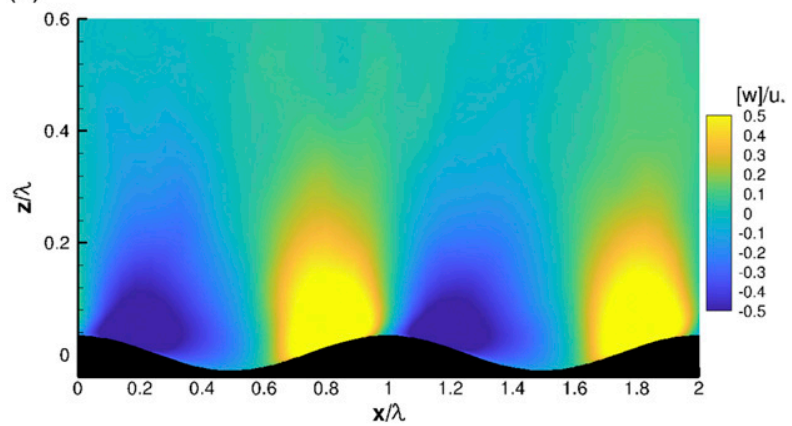

(b)

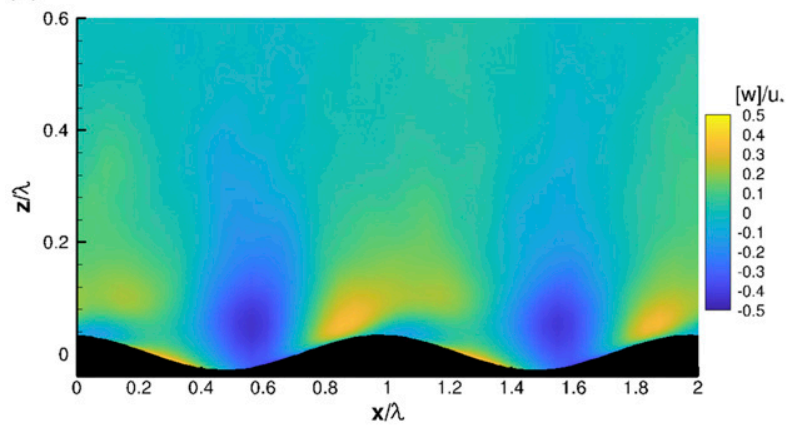

(c)

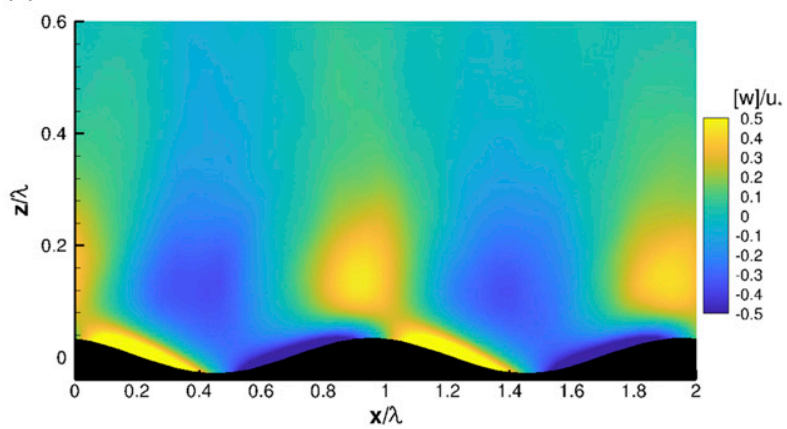

FIG. 2. Phase-averaged contours of normalized vertical velocity $[w] / u_{*}$ : (a) $c / u_{*}=1$, (b) $c / u_{*}=5$, and (c) $c / u_{*}=10$. Coordinates $z$ and $x$ are normalized by wavelength $\lambda$.

Marchioli et al. (2006), have described in detail the interaction of particles with coherent structures near the wavy surface, as well as the importance of the particle Stokes number on their spatial distribution and deposition. Marchioli et al. (2006) reports a significantly enhanced deposition rate of particles over a wavy surface compared to a flat surface, and this rate is highly sensitive to particle inertia. This enhanced deposition rate indicates that the vertical distribution of droplets suspended over a wavy surface will deviate from traditional models for vertical suspension.

\section{1) VERTICAL PROFILES OF CONCENTRATION}

Under equilibrium conditions, where spray emission from the surface balances deposition (i.e., zero net vertical flux), it can be shown that the average concentration varies as a power law with height (Rouse 1937; Prandtl 1952):

$$
\frac{\bar{C}(z)}{C_{r}}=\left(\frac{z}{z_{r}}\right)^{-w_{s} / \kappa u_{*}},
$$

where $\bar{C}(z)$ is the mean droplet concentration averaged in time and over the horizontal plane, $C_{r}$ is a reference concentration at height $z_{r}$, and $\kappa$ is the von Kármán constant (taken as $\kappa=0.41$ in this study). This expression is based on the assumption that turbulent transport can be described using a Monin-Obukhov eddy diffusivity $K_{C}=\kappa u * z$, which is not necessarily the case for inertial particles (Richter and Chamecki 2018). Equation (9) can be extended to include nonzero net fluxes (Kind 1992; Hoppel et al. 2002), unstable stratification (Freire et al. 2016), or 2D effects (Pan et al. 2013); however, the core of the present study focuses on the effects of droplet size and the wavy surface on the equilibrium concentration.

Since Eq. (9) is derived for an unbounded domain, a slight modification taking into account the upper no-flux boundary condition in our simulations must be made in order to compare the two. Here, the linear eddy diffusivity $K_{C}$ is replaced by a parabolic form representing a bounded domain (see, e.g., Fischer 1973):

$$
K_{C}=\kappa u_{*} z\left(1-\frac{z}{L_{z}}\right) .
$$

Instead of Eq. (9), this yields an expression for $\bar{C}(z)$, which follows

$$
\frac{\bar{C}(z)}{C_{r}}=\left(\frac{z}{z_{r}}\right)^{-w_{s} / \kappa u *}\left(\frac{z-L_{z}}{z_{r}-L_{z}}\right)^{w_{s} / \kappa u *},
$$

but is based on exactly the same assumption of equilibrium between emission and deposition.

Figure 4 plots the horizontally averaged, normalized mass concentration $\bar{C}(z) / C_{0}$ as a function of $z / \lambda$, where $C_{0}$ is the bulk concentration in the domain (total mass of droplets divided by the total domain volume). The simulation results are compared directly to the prediction of Eq. (10), where we use $z_{r} / \lambda=H_{d}$ since it is the maximum theoretical height that a droplet can reach without turbulent transport. This location $z_{r}$ is akin to the droplet source height described for example by Veron (2015) or Fairall et al. (2009).

As expected, the largest droplets tend to remain near the lower surface, whereas the smallest droplets tend to distribute nearly evenly across the vertical extent of the domain as would a passive scalar-a feature also seen qualitatively in the numerical models of 
(a)
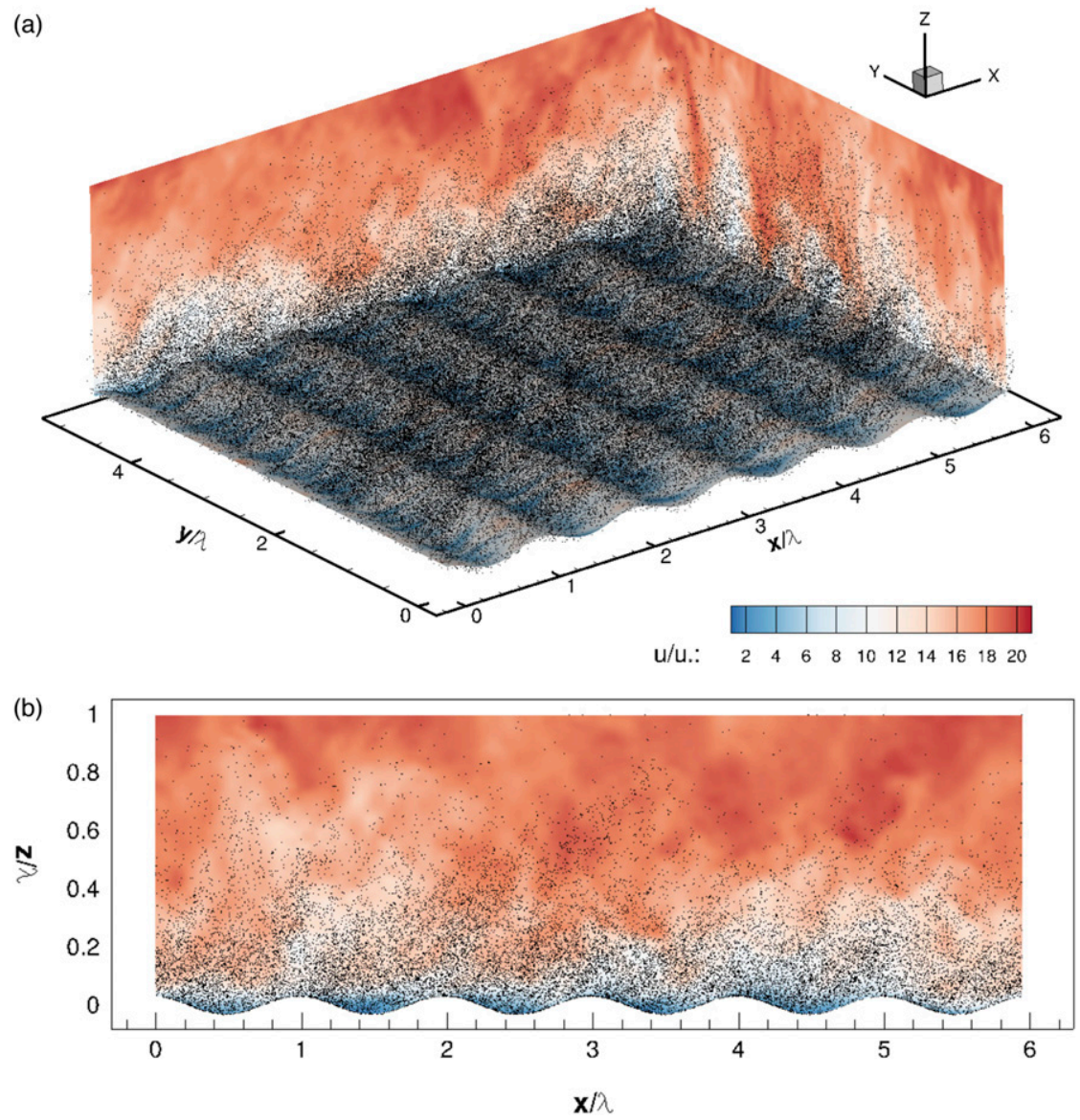

FIG. 3. Instantaneous snapshot of normalized streamwise velocity $u / u_{*}$ (color contours) over the wavy surface for case $\mathrm{c} 1\left(c / u_{*}=1, d_{p}=100 \mu \mathrm{m}\right)$. Black dots represent the instantaneous location of all particles in the domain. (a) Isometric view and (b) side view.

Edson and Fairall (1994), Druzhinin et al. (2017), and Tang et al. (2017). Likewise, laboratory experiments have observed the same vertical sorting of droplet sizes. The experiments of Mestayer and Lefauconnier (1988), which are able to directly study the transport of bubbleproduced jet and film droplets, show a clear dependence of the heights achieved by droplets on their size (in addition to turbulence levels), as well as the rate at which they deposit downstream from the spray source. For droplets produced by breaking waves in high winds, laboratory measurements by Ortiz-Suslow et al. (2016) show a clear reduction in the number of lofted droplets as their size increases. In the present simulations, the largest droplets $\left(d_{p}=600 \mu \mathrm{m}\right.$; Fig. $\left.4 \mathrm{f}\right)$ are confined essentially to a region below the height they could theoretically reach based on their initial injection velocity. Beneath the wave crest height (dashed horizontal lines), the concentration decreases with height as droplets become more and more susceptible to being overtaken by the wave and removed from the system.
The present analysis is designed to calculate statistics only when the droplets are truly in equilibrium - that is, statistics are calculated only after the concentration profile stops evolving in time when emission balances deposition. In this case, Eq. (10) would theoretically hold, and Fig. 4 indicates that it does, however only for small droplets and at sufficient heights above the wave. In Figs. $4 \mathrm{a}$ and $4 \mathrm{~b}$, corresponding to $d_{p}=10 \mu \mathrm{m}$ and $d_{p}=20 \mu \mathrm{m}$, the profiles are nearly vertical, and the low settling velocity $w_{s}$ results in a good agreement between the simulations and Eq. (10). With increasing $d_{p}$, however, there are two reasons for the discrepancy between the power-law profile and the simulations, particularly noticeable in Figs. 4d and 4e.

The first is due to droplet inertia, where droplets can depart from fluid streamlines, violating the assumptions behind Eq. (10)—namely, that the droplets travel exactly with the fluid velocity except for a constant setting velocity in the $z$ direction. Richter and Chamecki (2018) investigated this effect, and demonstrated that this is 
(a)

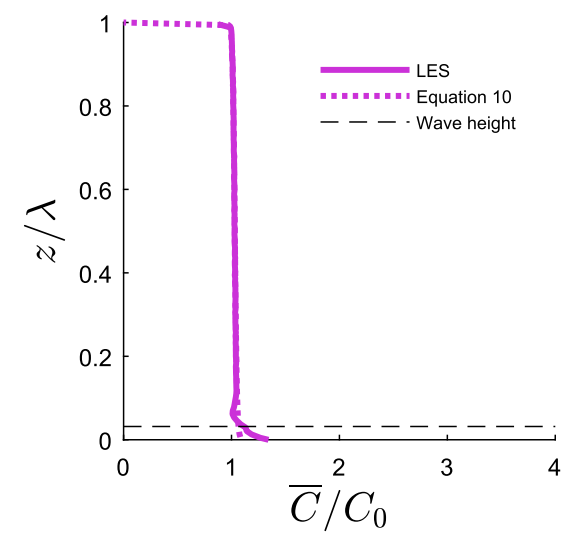

(c)

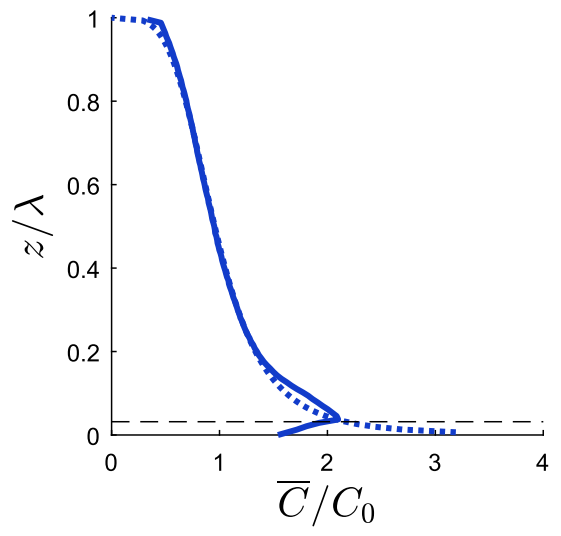

(e)

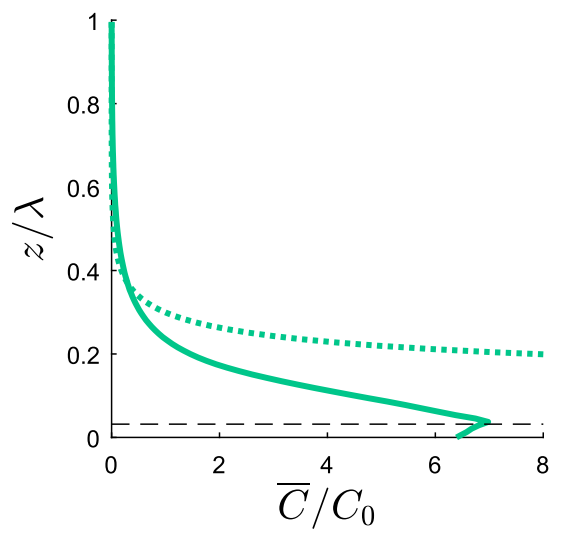

(b)

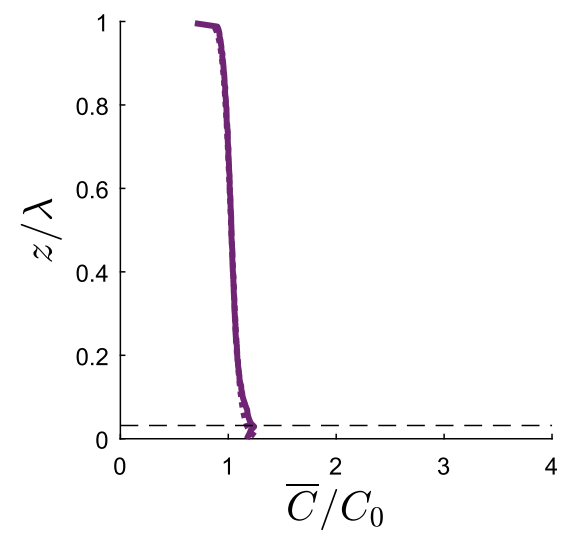

(d)

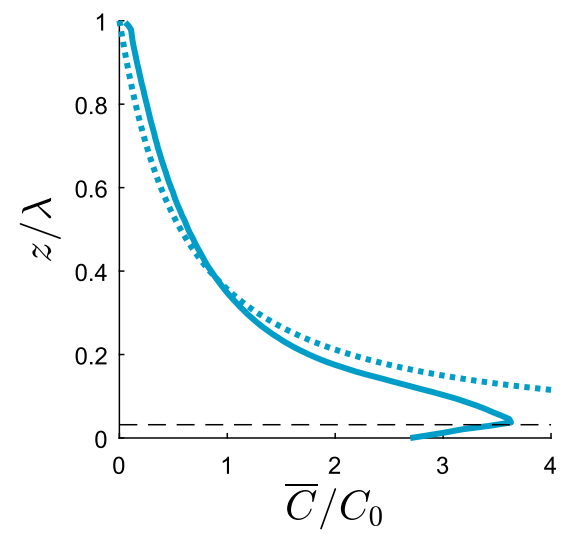

(f)

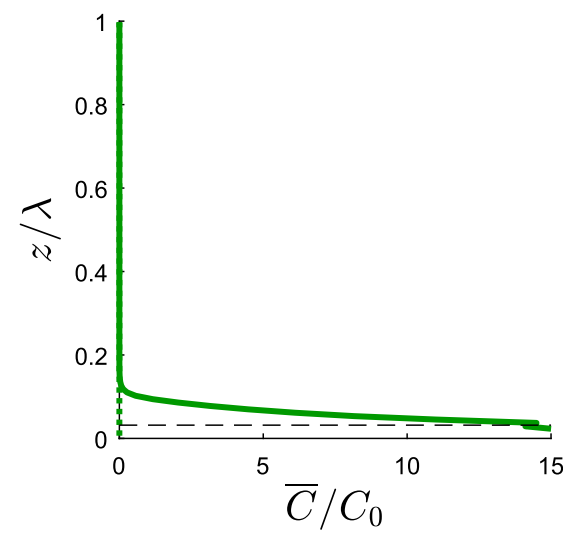

FIG. 4. Profiles of the horizontally averaged concentration $\bar{C}(z) / C_{0}$ plotted for the various droplet sizes, where $C_{0}$ is the bulk concentration. Note that with this normalization, the integral of each profile is the same across all $d_{p}$. Cases (a) d10, (b) d20, (c) d50, (d) d100, (e) d200, (f) and d600. Dotted lines show the prediction of Eq. (10) with $z_{r} / \lambda=0.36$. The thin horizontal dashed lines indicate the height of the wave crest.

related to the fact that inertial droplets do not experience the same eddy diffusivity as passive scalars. Figure 5 plots the average fluid velocity and average droplet velocity with height, and clearly demonstrates the increase of the slip velocity between the two as the droplet size grows-a feature seen for example in the experiments of Fairall et al. (2009) yet often neglected.

The second reason is due to the presence of the waves and the wave-induced turbulence-another effect neglected in the development of Eq. (10). Only at a height 


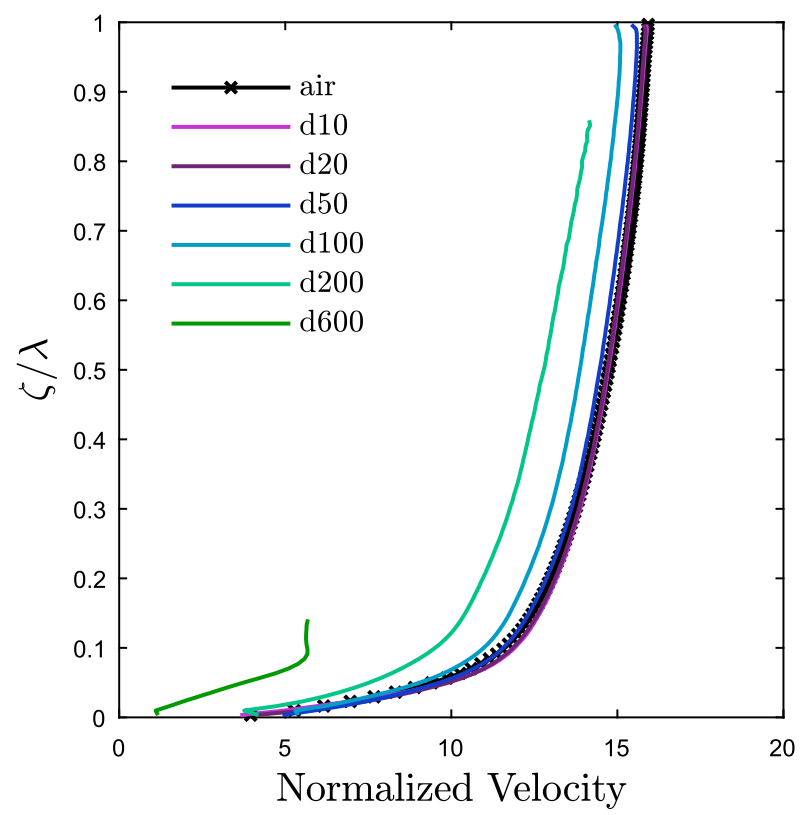

FIG. 5. Profiles of normalized air velocity $\langle u\rangle / u_{*}$ (symbols) and mean droplet velocities $\left\langle v_{p, 1}\right\rangle / u_{*}$ (solid lines) plotted against $\zeta / \lambda$. Colors refer to different droplet sizes, corresponding to the legend.

several times that of the wave amplitude (up to nearly a quarter or half the wavelength) do Eq. (10) and the LES agree, even for relatively inertialess droplets (Fig. 4c). In analogy with other types of surface roughness (canopies, topography, etc.) this is somewhat expected, since only at heights which scale with $z / h$, where $h$ is a characteristic roughness length should one expect to see logarithmic or self-similar scaling of momentum or scalars. So in effect, Fig. 4 suggests that Eq. (10) could in fact hold, but only at heights which increase with droplet radius due to their increasing inertia. The influence of wave-induced turbulence will be further investigated in section $3 \mathrm{c}$.

\section{2) WAVE-RELATIVE DROPLET BEHAVIOR}

To better understand how the waves modify droplet transport, Fig. 6 shows phase-averaged concentrations for cases d10-d600. The figure shows a clear, rapid transition as droplets get larger. For the smallest droplets, $d=10 \mu \mathrm{m}$ (Fig. 6a), there is a slight preference for higher droplet concentrations near the surface within the trough. This is primarily due to these small droplets never being transported above the wave and reentering the water almost immediately - this effect actually goes away with slightly larger droplets (Fig. 6b), since $20 \mu \mathrm{m}$ droplets possess slightly more inertia, which allows them to better sample the near-surface turbulence and therefore be better mixed (i.e., a subset of the $10 \mu \mathrm{m}$ droplets decelerate so quickly after injection that they immediately fall back into the water without being exposed to turbulent fluctuations). These two sizes correspond to the profiles in Fig. 4 that are nearly uniform with height above the wave. Figures $6 c$ and $6 d$ represent transitional cases, where droplets are heavy and inertial enough to yield higher concentrations near the surface, but are strongly influenced by wave-relative flows. Despite droplets being emitted uniformly along the surface in these simulations, the highest droplet concentrations lie in a clear band that connects the crest of a wave with the windward side of the downstream wave. This pattern is highly consistent with the photographic images and conceptual picture of Komori et al. (2018), who observed a distinct layer of droplet concentration in their wind-wave laboratory at high winds.

At still higher droplet sizes, Figs. $6 e$ and $6 \mathrm{f}$ show that droplets are mostly confined to the region near the wave, and only some escape into the air above. In particular, Fig. 6f suggests that only droplets emitted from the crest are carried above the maximum injection height (the maximum height the droplets could possibly reach with no ambient flow). Again, this transition between uniformly distributed droplets to those confined to the droplet ejection layer has been observed qualitatively in measurements (Ortiz-Suslow et al. 2016) and numerical models (Druzhinin et al. 2017; Edson and Fairall 1994). While the simulations herein were not designed to study the effects of intermittent wave breaking and the resulting heterogeneous droplet source locations (e.g., Lenain and Melville 2017), they do highlight the fact that waveinduced turbulence strongly influences their spatial distribution both in the horizontal and vertical directions, even when emission is homogeneous, and that the magnitude of this influence transitions with droplet size. We emphasize, however, that the uniform along-wave distribution seen, for example, in Fig. 6f is due to the imposed uniform droplet emission used in this study, and would clearly differ qualitatively for a more realistic emission strategy.

To further quantify this transition, Figs. 7 and 8 provide Lagrangian statistics of droplets from two representative cases: $\mathrm{d} 10$ and $\mathrm{d} 100$, respectively. In each of these figures, the fate of the droplets is described as a series of joint probability distributions (JPDFs). The first JPDF (Figs. 7a, 8a) is between the droplet initial $\left(x_{0}\right)$ and final $\left(x_{d}\right)$ wave-relative positions. The next (Figs. 7b, 8b) shows the JPDF between $x_{0}$ and the maximum height achieved during its lifetime $z_{\max }$, and the last JPDF (Figs. 7c, 8c) shows the relationship between $x_{0}$ and the droplet lifetime $t_{L}$.

Figure $7 \mathrm{a}$ shows that $d_{p}=10 \mu \mathrm{m}$ droplets, which according to Fig. $4 \mathrm{~b}$ distribute nearly uniformly in the verti$\mathrm{cal}$, are most likely to reenter the water just downstream of their emission location, particularly along the windward side of the wave (though this effect is relatively weak). 
(a)

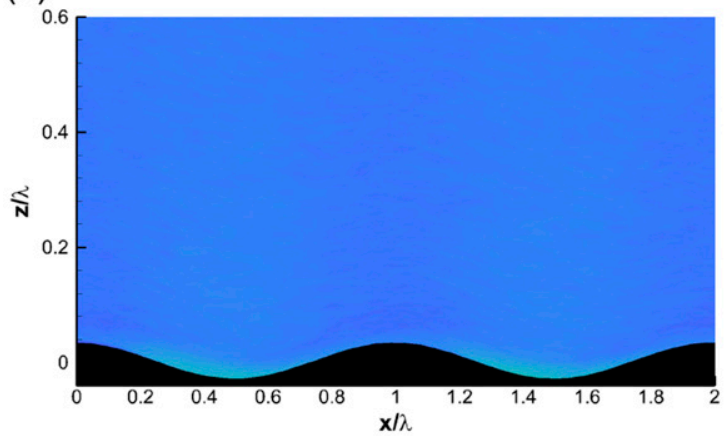

(c)

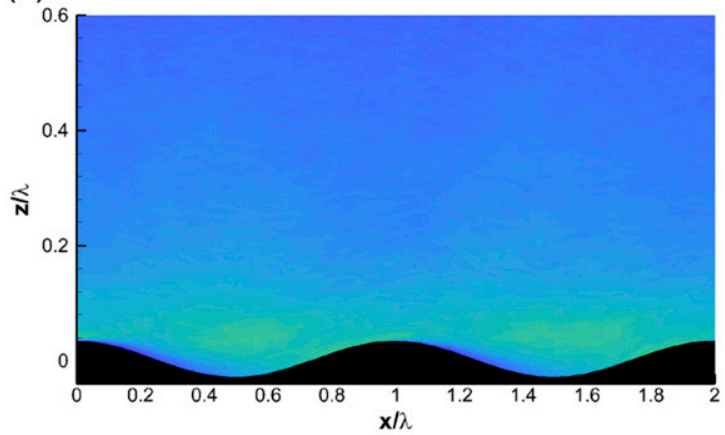

(e)

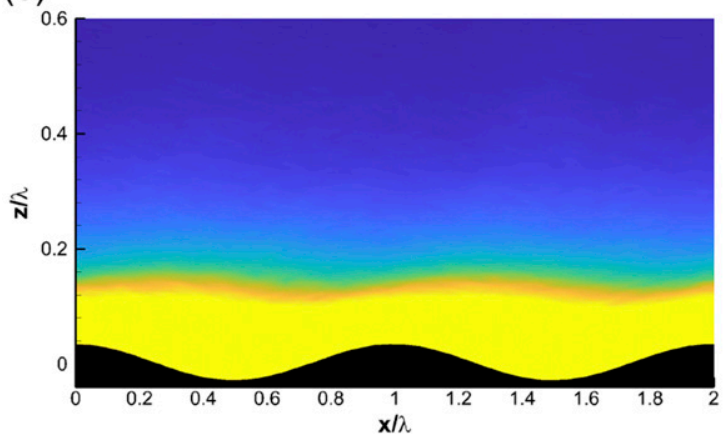

(b)

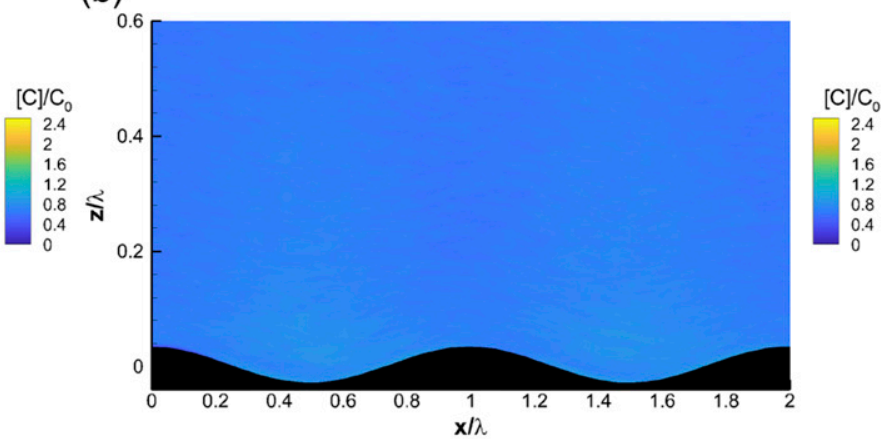

(d)

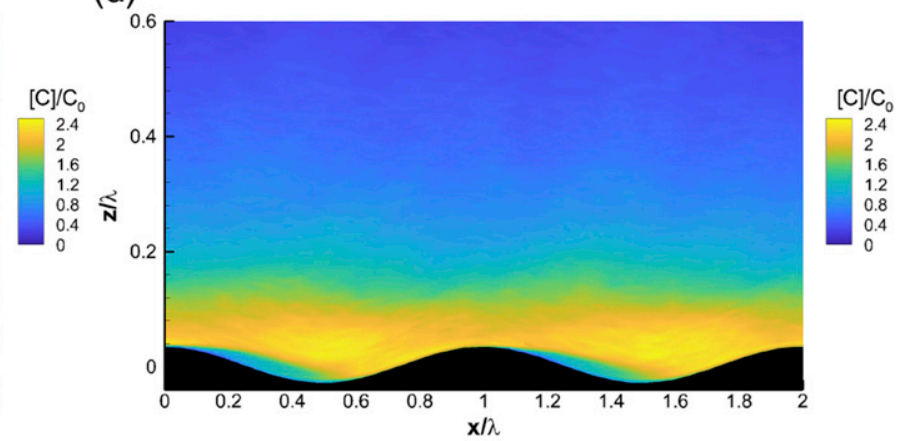

(f)

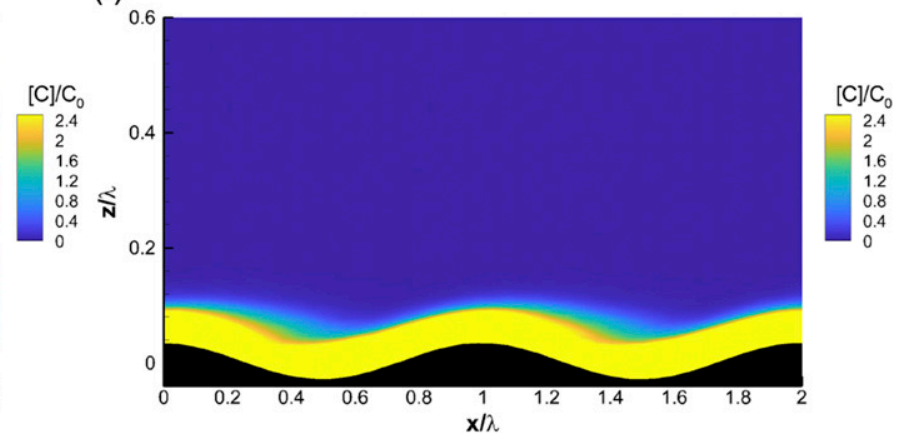

FIG. 6. Normalized phase-averaged concentration $[C] / C_{0}$ as a function of height $z / \lambda$ and $x / \lambda$ : (a) d10, (b) d20, (c) d50, (d) d100, (e) d200, and (f) d600.

Figure $7 \mathrm{~b}$ then illustrates that these small droplets generally have one of two fates. The first is that droplets immediately reenter the water because their low inertia retains them near the surface. They are immediately swept into the near-surface air streamline, and thus trapped, are susceptible to falling back into the water via their gravitational settling. The second is that the droplets work their way into the bulk flow and ultimately reach the top of the domain during their lifetime. This is corroborated by Fig. $7 \mathrm{c}$, which has a strong probability band at very short times (yellow stripe along the bottom), followed by a much broader range of longer-lived lifetimes. Neither the maximum height nor the lifetime of these small droplets is strongly influenced by the initial ejection location, except perhaps that droplets emerging from the trough of the wave are less likely overall to live as long or travel as high as those emitted from other parts of the wave. These droplets in the trough which have short lifetimes are also those which are seen in Fig. 7a to preferentially reenter downstream of their emission location.

In contrast, Fig. 8 shows that $d_{p}=100 \mu \mathrm{m}$ droplets exhibit significantly different behavior, due entirely to their increased inertia and settling velocity. Here, again two different droplet lifetime regimes emerge, however for distinct reasons than for the $d_{p}=10 \mu \mathrm{m}$ droplets. Figure $8 \mathrm{a}$ shows that many of the droplets, especially those emitted from the lee side of the wave, are immediately overtaken by the wave and reenter at the same or even slightly upstream wave-relative location. These are associated with very short lifetimes and low values of $z_{\max }$. Here, 
(a)

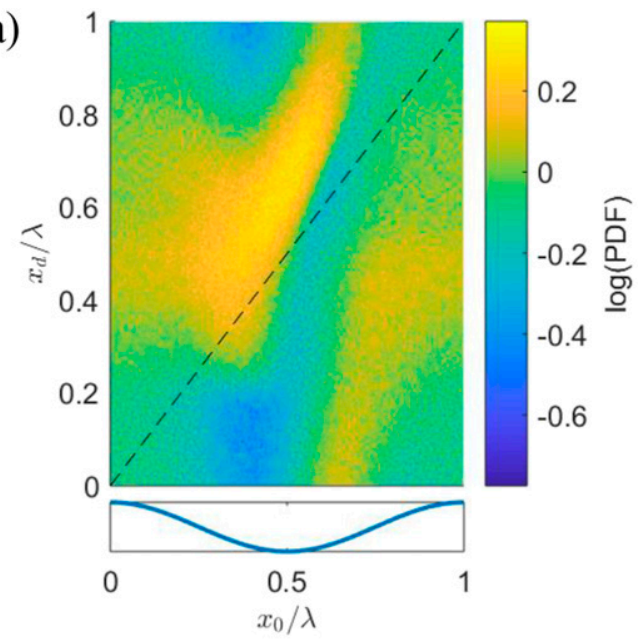

(b)

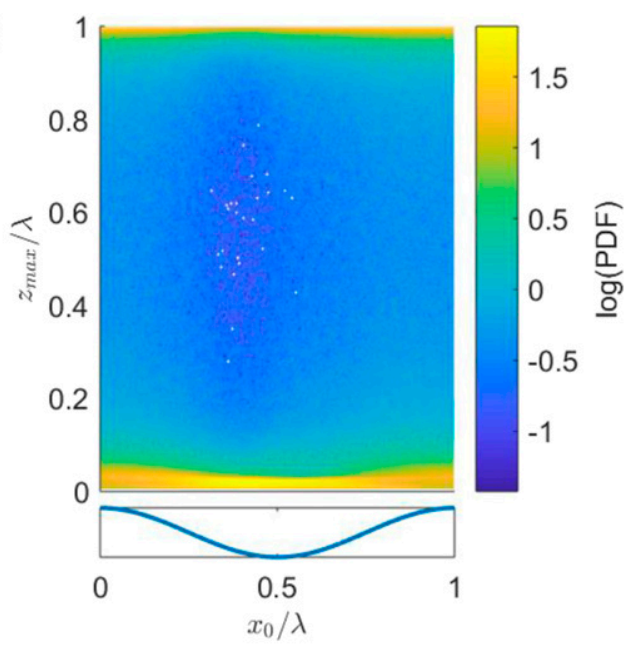

(c)

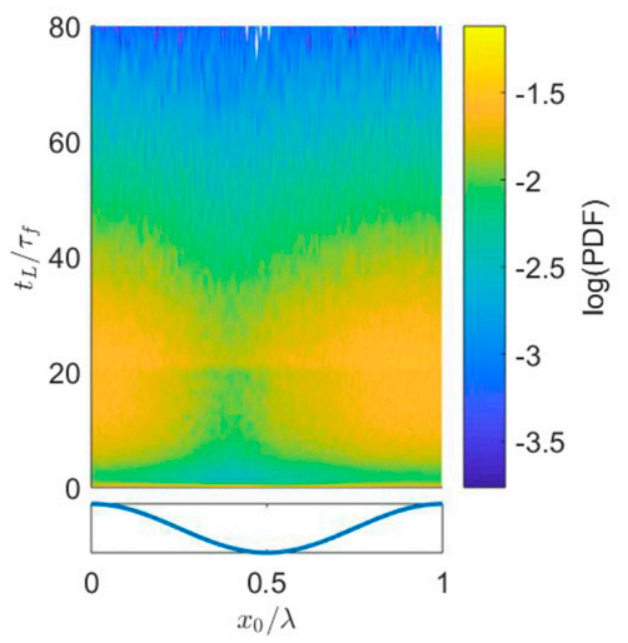

FIG. 7. Joint probability distributions of the droplet ejection location $x_{d}$ with (a) the droplet death location $x_{d}$ (dashed line is the 1:1 line, where droplets reenter at the same location as their initiation), (b) the maximum height reached by the droplet during its lifetime $z_{\max }$, and (c) the droplet lifetime $t_{L}$. The waveform in the lower panel is a schematic for referencing the position $x_{0}$. Case $\mathrm{d} 10$.

the droplet emerges with a velocity that is mostly vertical (its horizontal velocity is equal to that of the wave orbital velocity at that location), and its larger inertia prevents it from accelerating to the surrounding air velocity. Thus in a short period of time, the streamwise distance traveled by the droplet is actually less than that of the wave, and the droplet reenters the water as it is overtaken if it does not rise above the crest height. If droplets escape this fate, however, they live for a generally shorter amount of time than the smaller droplets (as expected), reach generally lower heights within the domain, and their reentry point is almost universally on the windward side of the downstream wave, consistent with Fig. 6d. Essentially no droplets reenter on the leeward side of the crest.

Ultimately, the picture that develops is as follows. The influence of wave-induced turbulence becomes stronger with droplet size, primarily because gravity and inertia prevent droplets from escaping the wave boundary layer. Small droplets really only feel the wave if they are ejected from the lee side, particularly near the trough, but even then many of these droplets are available for transport throughout the domain. As droplet diameter increases beyond $d_{p}=100 \mu \mathrm{m}$, the initial ejection location becomes much more important. Droplets emerging from the wave crests are more likely to be carried into the bulk flow, and all droplets, regardless of their initial location, are propelled into the windward face of waves. The implications of these distinct behaviors on spray-induced heat and momentum transfer are high, since in spray models it is often assumed that these droplets have lifetimes proportional to the time it takes to gravitationally settle from a significant wave height (e.g., Andreas et al. 2015). 
(a)

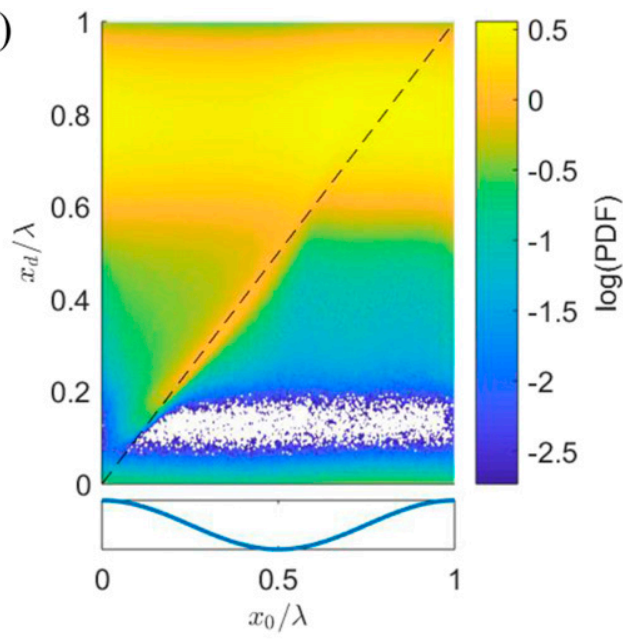

(b)

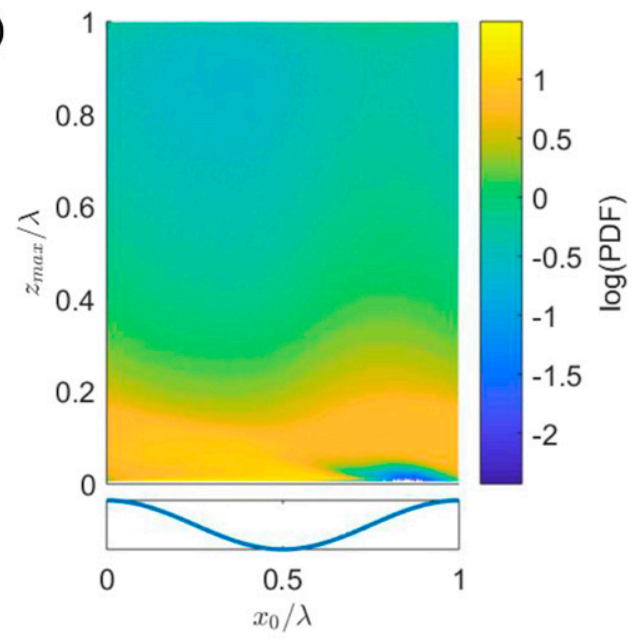

(c)

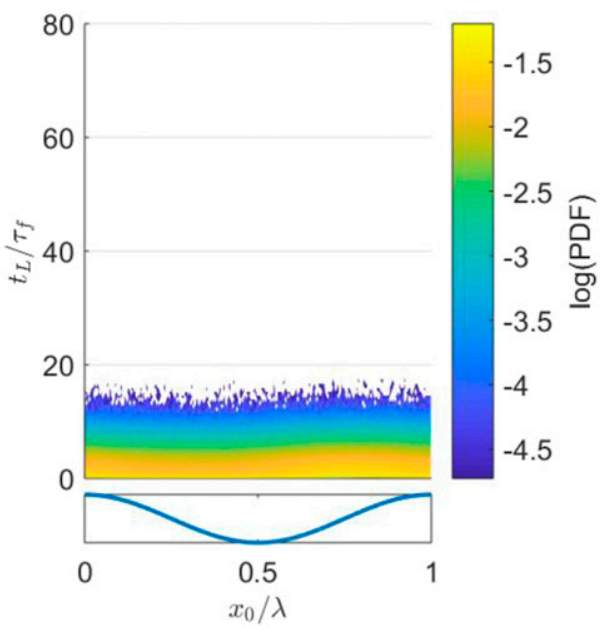

FIG. 8. As in Fig. 7, but for case d100.

The wave-induced deviation from the Prandtl powerlaw concentration profile likewise becomes more pronounced with droplet size, and also has its own implications on inferring spray source functions-this will be further discussed below in section 4 . Other factors, such as the initial droplet velocity, are likely important as well [see, e.g., the discussion in Druzhinin et al. (2017)], however the absence of firm observational data precludes a clear alternative to the idealized strategy chosen here. In the next section, we shift our focus to the effects of wave age on the droplet transport, and further discuss the implications of assuming a power-law distribution to infer spray source functions.

\section{3) Droplet statistics: EfFect of WAVE Age}

Figure 4 presents concentration profiles for varying droplet radii, each with the exact same wave/flow. To instead highlight the effect of wave-induced turbulence on the predictive capabilities of Eq. (10), Fig. 9 shows the same profiles, except while increasing wave age $c / u *$ and keeping the droplet Stokes number and settling velocity identical to that corresponding to the $d_{p}=100 \mu \mathrm{m}$ case. As a baseline, Fig. 9a shows the same profile for a flat lower surface, and demonstrates that the power-law profile very accurately predicts the LES results when no surface wave is present, at least above $z / \lambda \approx 0.05$. With the addition of a moving surface wave, increasing $c / u *$ from 1 to 10 leads to a larger and larger region near the surface where Eq. (10) is violated. The turbulence induced by the waves in this range of $c / u *$ becomes more effective at vertically mixing droplets in the vicinity of the wave, and the vertical extent of this region is in qualitative agreement with the region of wave-coherent vertical velocities shown in Fig. 2.

To confirm this, Fig. 10 shows the evolution of the phase-averaged concentration with wave age for $d_{p}=100 \mu \mathrm{m}$ droplets, along with the corresponding JPDF of droplet initial and final locations. Figures 10a 
(a)

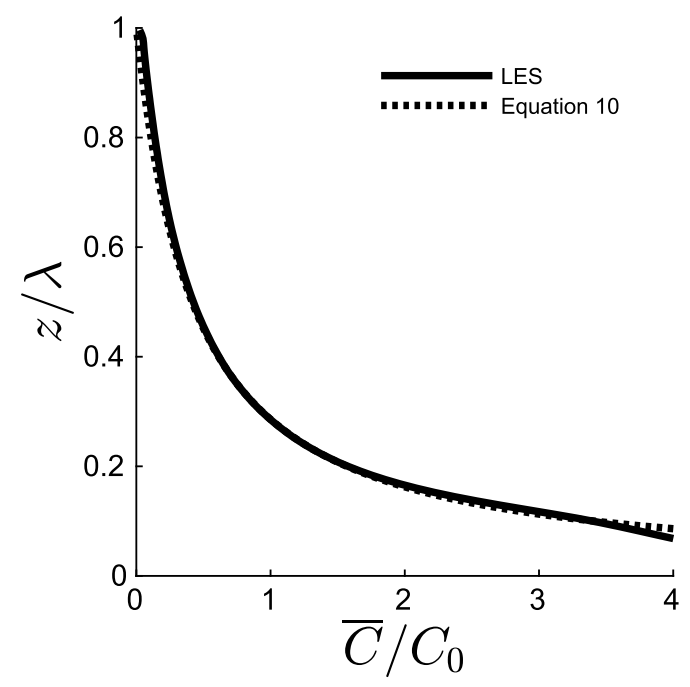

(c)

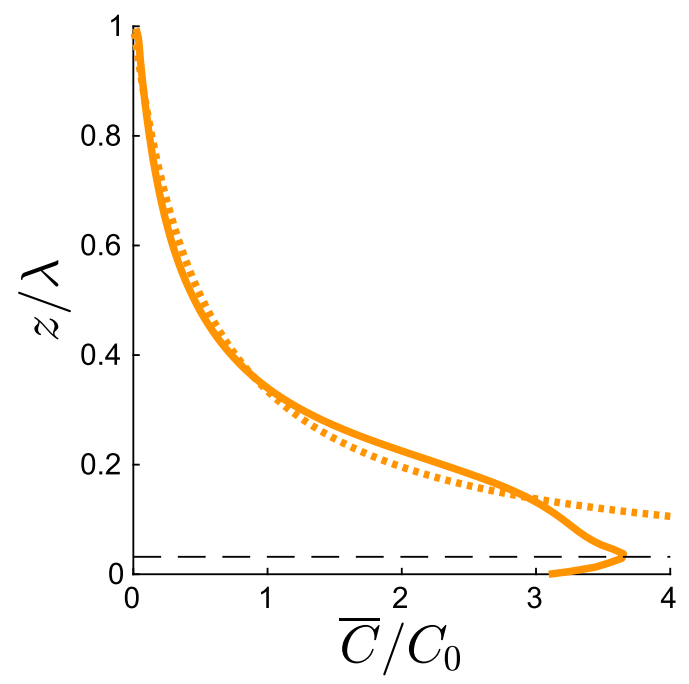

(b)

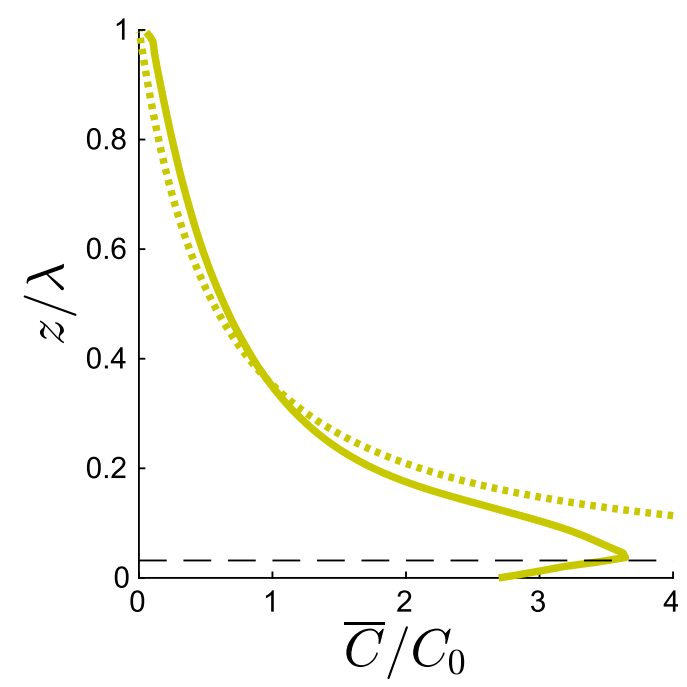

(d)

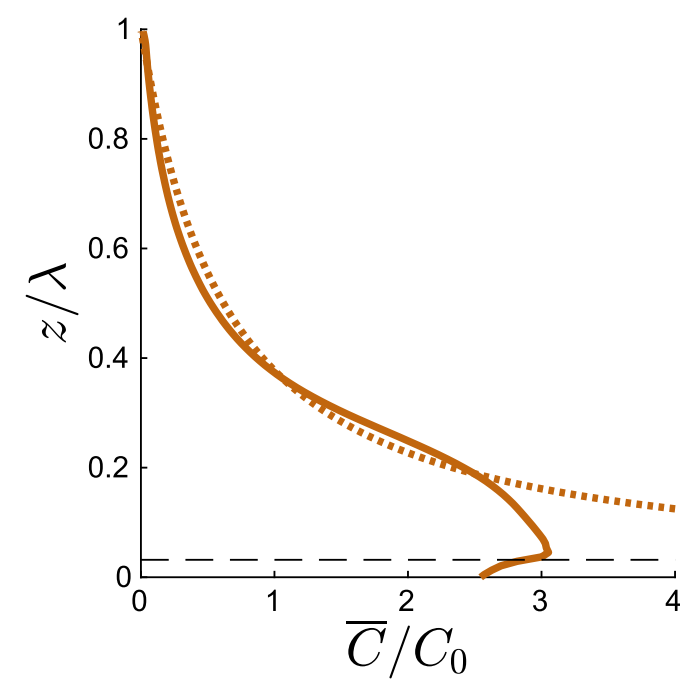

FIG. 9. As in Fig. 4, but for cases (a) f1, (b) c1, (c) c5, and (d) c10, highlighting concentration profiles for the same droplet Stokes number at different wave speeds.

and $10 \mathrm{~b}$ are the same as Figs. $6 \mathrm{~d}$ and $8 \mathrm{a}$, respectively, and are provided for reference. As the wave age increases above $c / u_{*}=1$, the high concentration band connecting the crest of a wave to the windward side of the downstream wave is disrupted (Figs. 10c,e). From Figs. 10d and 10f, it is clear that the reason for this is that many of the droplets are now being overtaken by the wave, particularly those emitted from the lee side. So while at $c / u_{*}=1$ the wind blows the ejected droplets in the streamwise direction and into the face of the downstream wave, at higher wave ages they are not accelerated sufficiently quickly to avoid being overtaken. By $c / u *=10$, the highest concentrations are actually found on the windward side of wave (as opposed to the lee side for $c / u_{*}=5$ ), and the positive phase-average vertical velocity above the wave crest (Fig. 2) leads to a more uniform vertical distribution of droplet concentration within the wave boundary layer.

There are strong implications, therefore, about the fate of droplets produced by different mechanisms. Small droplets, typically created by bubble bursting (e.g., jet and film droplets), are generally available for transport no matter where along the wave they are generated. Many of these low-inertia particles will be taken straight back into the water, while many will also be transported vertically. As the droplet size grows larger, however, only certain types of 
(a)

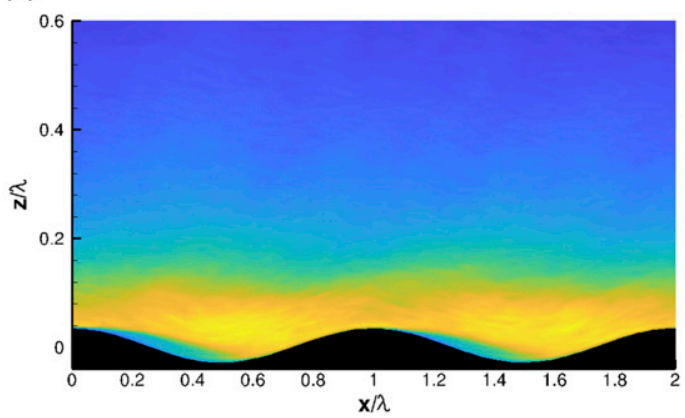

(c)

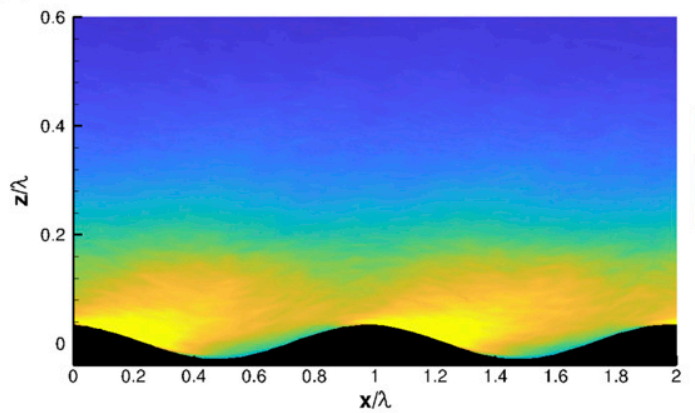

(e)

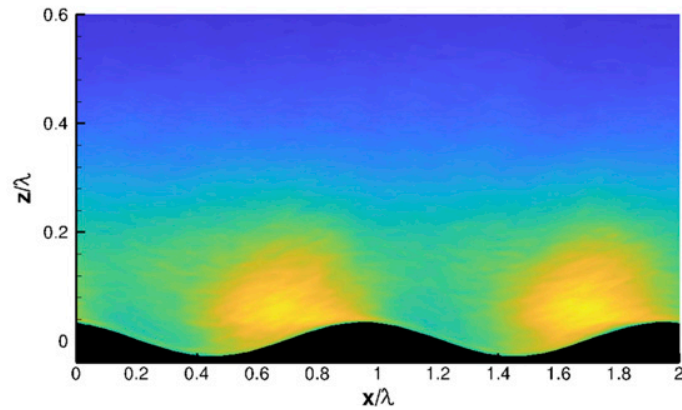

(b)
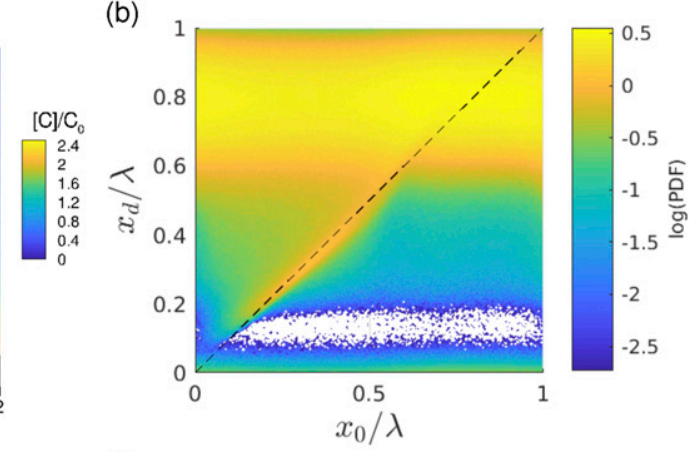

(d)

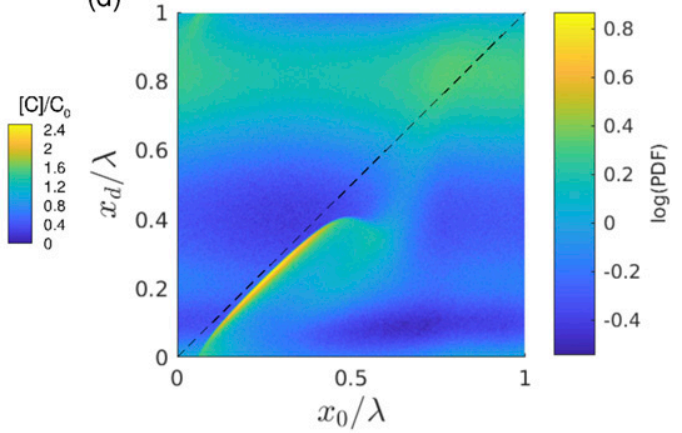

(f)

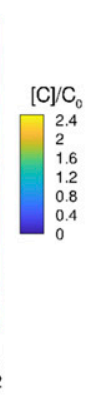

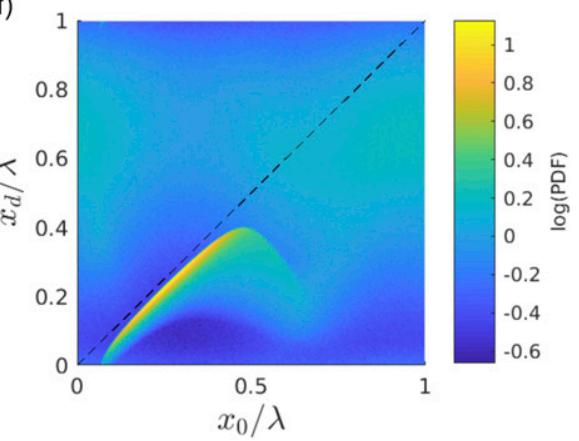

FIG. 10. (left) Normalized phase-averaged concentration $[C] / C_{0}$ as a function of height $z / \lambda$ and $x / \lambda$. (right) JPDF of droplet initial location $x_{0}$ and droplet final location $x_{d}$ : (a),(b) c1; (c),(d) c5; and (e),(f) c10.

droplets may be available for transport above the wave boundary layer. Depending on the wave age, spume droplets, which are often considered to be torn from wave crests, may be the only droplets which can avoid either being overtaken by a nearby wave or propelled into a downstream wave. Droplets exceeding roughly $d_{p}=100 \mu \mathrm{m}$ produced by splashing or as jet droplets in the wave troughs are more unlikely to escape the wave boundary layer and may never exceed the significant wave height.

\section{Discussion}

One of the primary purposes of this study is to assess the applicability of Eq. (10) with realistic droplet sizes and under the influence of wave-induced turbulence. Figure 4 demonstrates that small droplets, in spite of the waves at the surface, distribute uniformly throughout the vertical extent of the domain (above the wave crests), and as a result Eq. (10) accurately predicts their near-vertical concentration profile. As the droplet size increases, however, Eq. (10) becomes increasingly inaccurate due to a combination of wave effects and droplet inertia. Figure 9 further illustrates the influence of waves, highlighting that by simply changing the wave age, concentration profiles in the wave boundary layer can deviate significantly from a power law.

In practice, one of the more pertinent implications of this is in regards to the so-called flux-profile method for inferring source functions of spray and aerosols. With this method, mean concentrations are obtained at a fixed height, and the assumption is made that the deposition flux is in equilibrium with the upward turbulent flux. In 
(a)

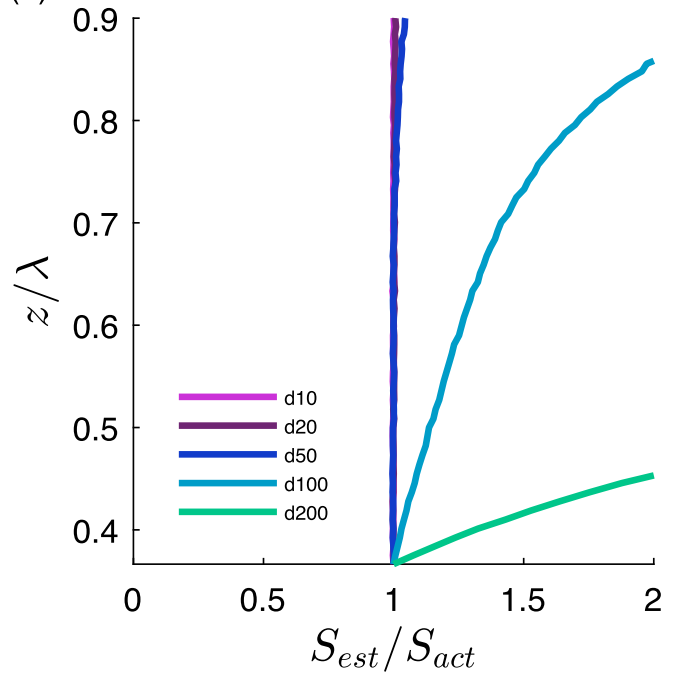

(b)

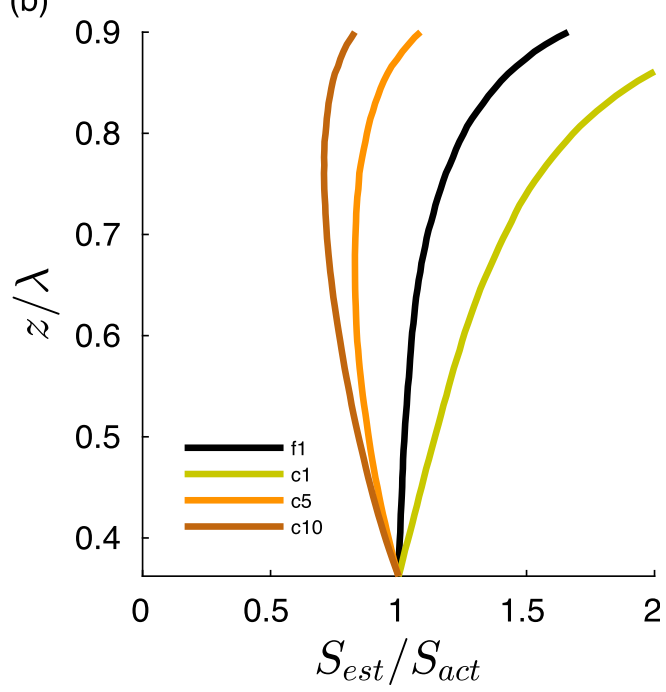

FIG. 11. The estimated $S_{\text {est }}$ normalized by the actual $S_{\text {act }}$ flux at height $z / \lambda=H_{d}$ as a function of $z / \lambda$. For all heights above $H_{d}$, the concentration at $H_{d}$ is estimated via Eq. (10), and the flux is approximated as $w_{s} \bar{C}$. (a) Varying droplet size for $c / u_{*}=1$ and (b) varying wave age for $d_{p}=100 \mu \mathrm{m}$.

this case, the flux at any height is assumed to be equal to $S(z)=V_{d} \bar{C}(z)$, where $V_{d}$ is a deposition velocity often assumed to be equal to the terminal settling velocity $w_{s}$. Therefore, if one assumes a concentration profile according to Eq. (10), the concentration $C_{r}$ measured at some height $z_{r}$ can be used to predict the concentration at some chosen source height, from which the source flux can be estimated. See, for example, the discussions in Veron (2015) or Fairall et al. (2009) for detailed discussions of this technique.

Indeed, experimental studies such as Fairall et al. (2009) and Ortiz-Suslow et al. (2016) use the collapse of the estimated surface fluxes measured from different measurement heights as an indicator of whether or not equilibrium has been established. In both of these studies, significant deviations between surface fluxes estimated from multiple heights were found, suggesting that the assumptions behind Eq. (10) (stationarity, equilibrium fluxes, logarithmic-law turbulence, etc.) were somehow violated. Fairall et al. (2009) actually see significant deviations for small droplet sizes, and interpret this to be evaporation effects in the wind tunnel (something not considered in this study). Ortiz-Suslow et al. (2016) observe a significant deviation at large droplet sizes. From our simulations, we argue that this is due to these large droplets being highly susceptible to both inertial effects and wave-induced turbulence effects. This is corroborated by looking at other experimental measurements, which take a more direct approach to estimating surface fluxes. For instance, Troitskaya et al. (2018a) use high-speed imaging to directly count droplets produced by the breakup of filaments, bubbles, and so-called "bags" directly at the water surface. Their estimates of surface droplet fluxes are therefore more indicative of what was actually produced at the water surface, and tend to overestimate the flux-profile estimates of Ortiz-Suslow et al. (2016) for very large droplets while at the same time matching the measurements of Veron et al. (2012), which are also based on near-surface imaging (see Fig. 13 of Troitskaya et al. 2018a).

Further numerical evidence of this is seen in Fig. 11, where we estimate the droplet flux at $z / \lambda=H_{d}$ using Eq. (10) for all simulations. In doing so we are treating $H_{d}$ as akin to a droplet source layer where one would like to estimate the source fluxes. We then compare this estimated value to the known flux $S_{\text {act }}=w_{s} \bar{C}\left(H_{d}\right)$, which occurs at $H_{d}$ as measured from the true concentration at that height. Starting with droplet radius, Fig. 11a shows that for $d_{p}=10 \mu \mathrm{m}$ and $d_{p}=20 \mu \mathrm{m}$ droplets, the flux at $H_{d}$ can be very accurately predicted from measurements at any height above this in the domain, which is expected given the near-vertical concentration profiles seen in Fig. 4. With larger droplets, however, this ceases to be true. For $d_{p}=100 \mu \mathrm{m}$ and $d_{p}=200 \mu \mathrm{m}$ droplets, the accuracy of the source flux estimates decreases rapidly with height. For instance, with $d_{p}=100 \mu \mathrm{m}$ droplets, taking concentration measurements only $0.7 \lambda$ above the mean water surface would yield flux estimates that are $50 \%$ larger than the true fluxes at $H_{d}$.

By comparing Fig. 11a to Fig. 11b, it can be argued that the reason for this overestimation is primarily due 
to wave effects (at least for $d_{p}=100 \mu \mathrm{m}$ ), since beneath a certain height $(z / \lambda \approx 0.7)$ the equilibrium prediction would have worked for the flat case. Droplet inertia does still play a role, however. As wave age is increased, the overestimation of case d100 actually transitions to an underestimate, and with no surface wave at all, the estimates are accurate up to a height of $z / \lambda \approx 0.7$, where the cumulative influence of droplet inertia on the concentration profile begins to take effect (Richter and Chamecki 2018).

Given that the height $H_{d}$ is roughly 11 wave amplitudes above the water surface, this would all suggest that using an equilibrium approximation for the concentration profile is problematic in practice, especially where a significant wave boundary layer can be identified. Essentially these results indicate that if Eq. (10) is to be used, it must be applied entirely outside of the wave boundary layer-that is, both the measurement height, as well as the height where the fluxes are to be estimated, must lie above the region where turbulence is strongly influenced by the wave, which could be up to nearly 10 times the wave amplitude. To make matters worse, large droplets, even in the absence of significant wave effects, also deviate from a power-law behavior due to their inertia, and at the same time often do not even escape the wave boundary layer in the first place due to their gravitational settling. Therefore, the only regime in which one could potentially use Eq. (10) is for very small droplets, but it is well-known that these can take a very long time to establish equilibrium (Hoppel et al. 2002). We therefore conclude that the combined role of waveinduced turbulence and droplet inertia can easily explain the discrepancies which are continually found in large droplet source functions.

\section{Conclusions}

LES of flow over moving surface waves coupled with Lagrangian particle tracking is used to investigate the dynamics of spray droplets emitted from the water surface. In particular, we focus on how wave-induced turbulence and the inertia of large droplets cause deviations of the vertical concentration profiles from the often-assumed, equilibrium power-law shape. Idealized, monochromatic waves are considered at the lower surface, and statistics are taken after the flow and droplets reach a statistically steady state. The wave age $c / u *$ is varied, as is the droplet diameter $d_{p}$, and it is demonstrated that these factors alter both the wave-relative and vertical distributions of droplets. Small droplets $\left(d_{p} \leq 50 \mu \mathrm{m}\right)$ exhibit low inertia and therefore are able to maintain a nominally homogeneous distribution throughout the domain. As a result, the flux-profile method for estimating surface fluxes from elevated concentration measurements works well, even in the presence of surface waves. For larger droplets, however $\left(d_{p} \geq 100 \mu \mathrm{m}\right)$, the combined effect of wave-induced turbulence and droplet inertia causes significant deviations of the concentration profile from the power-law theory, which results in severe over or underpredictions in the surface source flux, depending on wave age. With increased wave age, inertial droplets are found in different locations relative to the wave (in a phase-averaged sense), and their modified trajectories result in vertical fluxes that do not correspond to those assumed in the power-law theory. Lagrangian statistics of droplet lifetimes, maximum heights, and reentry locations illustrate various fates of droplets, including immediate reentry of droplets emitted within the trough due to fast moving waves. Perhaps unsurprisingly, droplets emitted from the crest of waves (i.e., spume droplets) are the most likely to live longest and escape the wave boundary layer. Ultimately, it is concluded in this study that caution must be used when applying the flux-profile method in the wave boundary layer, since for large droplets errors in the surface source can be as large as $100 \%$, although assuming an equilibrium droplet distribution may be appropriate above $z=O(\lambda)$. In practice, however, this may actually preclude the use of this method for very large droplets, since they typically do not rise above the necessary $O(10 A)$ or $O(\lambda)$ above the surface due to their large gravitational settling velocity. Thus, the combined effect of inertia and wave-induced turbulence is likely the cause of the continued uncertainty in spray source functions for large droplets.

Acknowledgments. The authors would like to acknowledge NSF Grants OCE-1829515 and AGS-1429921 for financial support. The National Center for Atmospheric Research is supported by the NSF. The authors would also like to acknowledge the Notre Dame Center for Research Computing, who provided all computational resources and support. Data presented in this manuscript can be found at https://doi.org/10.7274/r0-m8br-n972.

\section{REFERENCES}

Andreas, E. L, J. B. Edson, E. C. Monahan, M. P. Rouault, and S. D. Smith, 1995: The spray contribution to net evaporation from the sea: A review of recent progress. Bound.-Layer Meteor., 72, 3-52, https://doi.org/10.1007/ BF00712389.

_ L. Mahrt, and D. Vickers, 2015: An improved bulk air-sea surface flux algorithm, including spray-mediated transfer. Quart. J. Roy. Meteor. Soc., 141, 642-654, https://doi.org/ 10.1002/qj.2424.

Balachandar, S., and J. K. Eaton, 2010: Turbulent dispersed multiphase flow. Annu. Rev. Fluid Mech., 42, 111-133, https:// doi.org/10.1146/annurev.fluid.010908.165243. 
Banner, M. L., 1990: The influence of wave breaking on the surface pressure distribution in wind-wave interactions. J. Fluid Mech., 211, 463-495, https://doi.org/10.1017/S0022112090001653.

Belcher, S. E., and J. C. R. Hunt, 1998: Turbulent flow over hills and waves. Annu. Rev. Fluid Mech., 30, 507-538, https:// doi.org/10.1146/annurev.fluid.30.1.507.

Blanchard, D. C., A. H. Woodcock, and R. J. Cipriano, 1984: The vertical distribution of the concentration of sea salt in the marine atmosphere near Hawaii. Tellus, 36B, 118-125, https:// doi.org/10.1111/j.1600-0889.1984.tb00233.x.

Buckles, J., T. J. Hanratty, and R. J. Adrian, 1984: Turbulent flow over large-amplitude wavy surfaces. J. Fluid Mech., 140, 2744, https://doi.org/10.1017/S0022112084000495.

Buckley, M. P., and F. Veron, 2016: Structure of the airflow above surface waves. J. Phys. Oceanogr., 46, 1377-1397, https:// doi.org/10.1175/JPO-D-15-0135.1.

De Angelis, V., P. Lombardi, and S. Banerjee, 1997: Direct numerical simulation of turbulent flow over a wavy wall. Phys. Fluids, 9, 2429, https://doi.org/10.1063/1.869363.

Deike, L., W. K. Melville, and S. Popinet, 2016: Air entrainment and bubble statistics in breaking waves. J. Fluid Mech., 801, 91-129, https://doi.org/10.1017/jfm.2016.372.

de Leeuw, G., 1986: Vertical profiles of giant particles close above the sea surface. Tellus, 38B, 51-61, https://doi.org/10.1111/ j.1600-0889.1986.tb00087.x.

— E. L Andreas, M. D. Anguelova, C. W. Fairall, E. R. Lewis, C. D. O'Dowd, M. Schulz, and S. E. Schwartz, 2011: Production flux of sea spray aerosol. Rev. Geophys., 49, RG2001, https://doi.org/10.1029/2010RG000349.

Derakhti, M., and J. T. Kirby, 2014: Bubble entrainment and liquidbubble interaction under unsteady breaking waves. J. Fluid Mech., 761, 464-506, https://doi.org/10.1017/jfm.2014.637.

Druzhinin, O. A., Y. I. Troitskaya, and S. S. Zilitinkevich, 2012: Direct numerical simulation of a turbulent wind over a wavy water surface. J. Geophys. Res., 117, C00J05, https://doi.org/ 10.1029/2011JC007789.

- —, and — 2017: The study of droplet-laden turbulent airflow over waved water surface by direct numerical simulation. J. Geophys. Res. Oceans, 122, 1789-1807, https://doi.org/ 10.1002/2016JC012134.

_ — - and — - 2018: The study of momentum, mass, and heat transfer in a droplet-laden turbulent airflow over a waved water surface by direct numerical simulation. J. Geophys. Res. Oceans, 123, 8346-8365, https://doi.org/ 10.1029/2018JC014346.

Edson, J. B., and C. W. Fairall, 1994: Spray droplet modeling: 1. Lagrangian model simulation of the turbulent transport of evaporating droplets. J. Geophys. Res., 99, 25 295-25311, https://doi.org/10.1029/94JC01883.

— , and Coauthors, 2013: On the exchange of momentum over the open ocean. J. Phys. Oceanogr., 43, 1589-1610, https:// doi.org/10.1175/JPO-D-12-0173.1.

Fairall, C. W., M. L. Banner, W. L. Peirson, W. Asher, and R. P. Morison, 2009: Investigation of the physical scaling of sea spray spume droplet production. J. Geophys. Res., 114, C10001, https://doi.org/10.1029/2008JC004918.

Fischer, H. B., 1973: Longitudinal dispersion and turbulent mixing in open-channel flow. Annu. Rev. Fluid Mech., 5, 59-78, https://doi.org/10.1146/annurev.fl.05.010173.000423.

Freire, L. S., M. Chamecki, and J. A. Gillies, 2016: Flux-profile relationship for dust concentration in the stratified atmospheric surface layer. Bound.-Layer Meteor., 160, 249-267, https://doi.org/10.1007/s10546-016-0140-2.
Grachev, A. A., and C. W. Fairall, 2001: Upward momentum transfer in the marine boundary layer. J. Phys. Oceanogr., 31, 1698-1711, https://doi.org/10.1175/1520-0485(2001)031<1698: UMTITM $>2.0 . \mathrm{CO} ; 2$.

Grare, L., L. Lenain, and W. Melville, 2013: Wave-coherent airflow and critical layers over ocean waves. J. Phys. Oceanogr., 43, 2156-2172, https://doi.org/10.1175/JPO-D-13-056.1.

Hara, T., and P. P. Sullivan, 2015: Wavy boundary layer turbulence over surface waves in a strongly forced condition. J. Phys. Oceanogr., 45, 868-883, https://doi.org/10.1175/JPOD-14-0116.1.

Helgans, B., and D. H. Richter, 2016: Turbulent latent and sensible heat flux in the presence of evaporative droplets. Int. J. Multiph. Flow, 78, 1-11, https://doi.org/10.1016/j.ijmultiphaseflow.2015.09.010.

Hoppel, W. A., G. M. Frick, and J. W. Fitzgerald, 2002: Surface source function for sea-salt aerosol and aerosol dry deposition to the ocean surface. J. Geophys. Res., 107, 4382, https:// doi.org/10.1029/2001JD002014.

Hristov, T. S., S. D. Miller, and C. A. Friehe, 2003: Dynamical coupling of wind and ocean waves through. Nature, 422, 55-58, https://doi.org/10.1038/nature01382.

Jiang, Q., P. P. Sullivan, S. Wang, J. Doyle, and L. Vincent, 2016: Impact of swell on air-sea momentum flux and marine boundary layer under low-wind condition. J. Atmos. Sci., 73, 2683-2697, https://doi.org/10.1175/JAS-D-15-0200.1.

Jones, K. F., and E. L Andreas, 2012: Sea spray concentrations and the icing of fixed offshore structures. Quart. J. Roy. Meteor. Soc., 138, 131-144, https://doi.org/10.1002/qj.897.

Kind, R. J., 1992: One-dimensional aeolian suspension above beds of loose particles - A new concentration-profile equation. Atmos. Environ., 26A, 927-931, https://doi.org/10.1016/09601686(92)90250-O.

Komori, S., K. Iwano, N. Takagaki, R. Onishi, R. Kurose, K. Takahashi, and N. Suzuki, 2018: Laboratory measurements of heat transfer and drag coefficients at extremely high wind speeds. J. Phys. Oceanogr., 48, 959-974, https://doi.org/10.1175/ JPO-D-17-0243.1.

Kudryavtsev, V. N., and V. K. Makin, 2001: The impact of air-flow separation on the drag of the sea surface. Bound.-Layer Meteor., 98, 155-171, https://doi.org/10.1023/A:1018719917275.

Lenain, L., and W. K. Melville, 2017: Evidence of sea-state dependence of aerosol concentration in the marine atmospheric boundary layer. J. Phys. Oceanogr., 47, 69-84, https://doi.org/ 10.1175/JPO-D-16-0058.1.

Lewis, E. R., and S. E. Schwartz, 2004: Sea Salt Aerosol Production: Mechanisms, Methods, Measurements, and Models: A Critical Review. Geophys. Monogr., Vol.152, Amer. Geophys. Union, 413 pp.

Marchioli, C., V. Armenio, M. V. Salvetti, and A. Soldati, 2006: Mechanisms for deposition and resuspension of heavy particles in turbulent flow over wavy interfaces. Phys. Fluids, 18 , 025102, https://doi.org/10.1063/1.2166453.

Mestayer, P., and C. Lefauconnier, 1988: Spray droplet generation, transport, and evaporation in a wind wave tunnel during the humidity exchange over the sea experiments in the simulation tunnel. J. Geophys. Res., 93, 572-586, https://doi.org/10.1029/ JC093iC01p00572.

Miles, J. W., 1957: On the generation of surface waves by shear flows. J. Fluid Mech., 3, 185-204, https://doi.org/10.1017/ S0022112057000567.

Moeng, C.-H., 1984: A large-eddy-simulation model for the study of planetary boundary-layer turbulence. J. Atmos. Sci., 41, 2052-2062, https://doi.org/10.1175/1520-0469(1984)041<2052: ALESMF $>2.0 . \mathrm{CO} ; 2$. 
Monahan, E. C., 1968: Sea spray as a function of low elevation wind speed. J. Geophys. Res., 73, 1127-1137, https://doi.org/10.1029/ JB073i004p01127.

Ortiz-Suslow, D. G., B. K. Haus, S. Mehta, and N. J. M. Laxague, 2016: Sea spray generation in very high winds. J. Atmos. Sci., 73, 3975-3995, https://doi.org/10.1175/JAS-D-15-0249.1.

Pan, Y., M. Chamecki, and S. A. Isard, 2013: Dispersion of heavy particles emitted from area sources in the unstable atmospheric boundary layer. Bound.-Layer Meteor., 146, 235-256, https://doi.org/10.1007/s10546-012-9753-2.

Peng, T., and D. Richter, 2017: Influence of evaporating droplets in the turbulent marine atmospheric boundary layer. Bound.-Layer Meteor., 165, 497-518, https://doi.org/10.1007/s10546-017-0285-7.

Prandtl, L., 1952: Essentials of Fluid Dynamics with Applications to Hydraulics, Aeronautics, Meteorology, and Other Subjects. Hafner Publishing Company, 452 pp.

Reul, N., H. Branger, and J. P. Giovanangeli, 2008: Air flow structure over short-gravity breaking water waves. Bound.-Layer Meteor., 126, 477-505, https://doi.org/10.1007/s10546-007-9240-3.

Richter, D. H., and P. P. Sullivan, 2013: Momentum transfer in a turbulent, particle-laden Couette flow. Phys. Fluids, 25, 053304, https://doi.org/10.1063/1.4804391.

— , and M. Chamecki, 2018: Inertial effects on the vertical transport of suspended particles in a turbulent boundary layer. Bound.-Layer Meteor., 167, 235-256, https://doi.org/10.1007/ s10546-017-0325-3.

Rouse, H., 1937: Modern conceptions of the mechanics of fluid turbulence. Trans. Amer. Soc. Civ. Eng., 102, 463-505.

Sullivan, P. P., and J. C. McWilliams, 2010: Dynamics of winds and currents coupled to surface waves. Annu. Rev. Fluid Mech., $\mathbf{4 2}$, 19-42, https://doi.org/10.1146/annurev-fluid-121108-145541.

— - — , and C.-H. Moeng, 1996: A grid nesting method for largeeddy simulation of planetary boundary-layer flows. Bound.-Layer Meteor., 80, 167-202, https://doi.org/10.1007/BF00119016.

,-- , and - , 2000: Simulation of turbulent flow over idealized water waves. J. Fluid Mech., 404, 47-85, https://doi.org/ 10.1017/S0022112099006965.

_ - J. B. Edson, T. Hristov, and J. C. McWilliams, 2008: Largeeddy simulations and observations of atmospheric marine boundary layers above nonequilibrium surface waves. J. Atmos. Sci., 65, 1225-1245, https://doi.org/10.1175/2007JAS2427.1.

_ J. C. McWilliams, and E. G. Patton, 2014: Large-eddy simulation of marine atmospheric boundary layers above a spectrum of moving waves. J. Atmos. Sci., 71, 4001-4027, https://doi.org/10.1175/JAS-D-14-0095.1.
— M. L. Banner, R. P. Morison, and W. L. Peirson, 2018a: Impacts of wave age on turbulent flow and drag of steep waves. Procedia IUTAM, 26, 174-183, https://doi.org/10.1016/ j.piutam.2018.03.017.

$\left[,-, \ldots\right.$, and $\_, 2018 \mathrm{~b}$ : Turbulent flow over steep steady and unsteady waves under strong wind forcing. J. Phys. Oceanogr., 48, 3-27, https://doi.org/10.1175/JPO-D-17-0118.1.

Tamura, H., W. M. Drennan, C. O. Collins, and H. C. Graber, 2018: Turbulent airflow and wave-induced stress over the ocean. Bound.-Layer Meteor., 169, 47-66, https://doi.org/10.1007/ s10546-018-0359-1.

Tang, S., Z. Yang, C. Liu, Y.-H. Dong, and L. Shen, 2017: Numerical study on the generation and transport of spume droplets in wind over breaking waves. Atmosphere, 8, 248, https://doi.org/10.3390/ atmos 8120248 .

Troitskaya, Y., A. Kandaurov, O. Ermakova, D. Kozlov, D. Sergeev, and S. Zilitinkevich, 2017: Bag-breakup fragmentation as the dominant mechanism of sea-spray production in high winds. Sci. Rep., 7, 1-4, https://doi.org/10.1038/s41598-01701673-9.

,,,,----- , and,$- 2018 \mathrm{a}$ : The "Bag Breakup" spume droplet generation mechanism at high winds. Part I: Spray generation function. J. Phys. Oceanogr., 48, 2167-2188, https://doi.org/10.1175/JPO-D-17-0104.1.

Troitskaya, Y. I., O. Druzhinin, D. Kozlov, and S. Zilitinkevich, 2018b: The "Bag Breakup" spume droplet generation mechanism at high winds. Part II: Contribution to momentum and enthalpy transfer. J. Phys. Oceanogr., 48, 2189-2207, https:// doi.org/10.1175/JPO-D-17-0105.1.

Veron, F., 2015: Ocean spray. Annu. Rev. Fluid Mech., 47, 507-538, https://doi.org/10.1146/annurev-fluid-010814-014651.

—, C. Hopkins, E. L. Harrison, and J. A. Mueller, 2012: Sea spray spume droplet production in high wind speeds. Geophys. Res. Lett., 39, L16602, https://doi.org/10.1029/2012GL052603.

Wanninkhof, R., W. E. Asher, D. T. Ho, C. S. Sweeney, and W. R. McGillis, 2009: Advances in quantifying air-sea gas exchange and environmental forcing. Annu. Rev. Mar. Sci., 1, 213-244, https://doi.org/10.1146/annurev.marine.010908.163742.

Weil, J. C., P. P. Sullivan, and C.-H. Moeng, 2004: The use of largeeddy simulations in Lagrangian particle dispersion models. J. Atmos. Sci., 61, 2877-2887, https://doi.org/10.1175/JAS3302.1 .

Yang, D., and L. Shen, 2010: Direct-simulation-based study of turbulent flow over various waving boundaries. J. Fluid Mech., 650, 131-180, https://doi.org/10.1017/S0022112009993557. 